\title{
Microbiome of Field Grown Hemp Reveals Potential Microbial Interactions With Root and Rhizosphere Soil
}

OPEN ACCESS

Edited by:

Wei Zhang,

Michigan State University,

United States

Reviewed by:

Anamika Dubey,

Dr. Hari Singh Gour University, India

Manoj Kumar Solanki,

University of Silesia in Katowice,

Poland

*Correspondence:

Mohamed Hijr

Mohamed.Hijri@umontreal.ca

Specialty section:

This article was submitted to

Terrestrial Microbiology,

a section of the journal

Frontiers in Microbiology

Received: 15 July 2021

Accepted: 11 October 2021

Published: 15 November 2021

Citation:

Ahmed B, Smart LB and Hijri $M$ (2021) Microbiome of Field Grown Hemp Reveals Potential Microbial Interactions With Root and Rhizosphere Soil.

Front. Microbiol. 12:741597. doi: 10.3389/fmicb.2021.741597

\section{Bulbul Ahmed ${ }^{1,2}$, Lawrence B. Smart ${ }^{2}$ and Mohamed Hijri $1^{1,3 *}$}

${ }^{1}$ Institut de Recherche en Biologie Végétale, Université de Montréal, Montréal, QC, Canada, ${ }^{2}$ Horticulture Section, School of Integrative Plant Science, Cornell AgriTech, Cornell University, Geneva, NY, United States, ${ }^{3}$ African Genome Center, Mohammed VI Polytechnic University (UM6P), Ben Guerir, Morocco

Hemp (Cannabis sativa L.) is a crop bred and grown for the production of fiber, grain, and floral extracts that contribute to health and wellness. Hemp plants interact with a myriad of microbiota inhabiting the phyllosphere, endosphere, rhizoplane, and rhizosphere. These microbes offer many ecological services, particularly those of below ground biotopes which are involved in nutrient cycling, uptake, and alleviating biotic and abiotic stress. The microbiota communities of the hemp rhizosphere in the field are not well documented. To discover core microbiota associated with field grown hemp, we cultivated single C. sativa cultivar, "TJ's CBD," in six different fields in New York and sampled hemp roots and their rhizospheric soil. We used Illumina MiSeq amplicon sequencing targeting 16S ribosomal DNA of bacteria and ITS of fungi to study microbial community structure of hemp roots and rhizospheres. We found that Planctobacteria and Ascomycota dominated the taxonomic composition of hemp associated microbial community. We identified potential core microbiota in each community (bacteria: eight bacterial amplicon sequence variant - ASV, identified as Gimesia maris, Pirellula sp. Lacipirellula limnantheis, Gemmata sp. and unclassified Planctobacteria; fungi: three ASVs identified as Fusarium oxysporum, Gibellulopsis piscis, and Mortierella minutissima). We found 14 ASVs as hub taxa [eight bacterial ASVs (BASV) in the root, and four bacterial and two fungal ASVs in the rhizosphere soil], and 10 BASV connected the root and rhizosphere soil microbiota to form an extended microbial communication in hemp. The only hub taxa detected in both the root and rhizosphere soil microbiota was ASV37 (Caulifigura coniformis), a bacterial taxon. The core microbiota and Network hub taxa can be studied further for biocontrol activities and functional investigations in the formulation of hemp bioinoculants. This study documented the microbial diversity and community structure of hemp grown in six fields, which could contribute toward the development of bioinoculants for hemp that could be used in organic farming.

Keywords: Cannabis sativa, rhizosphere, microbiome, bacterial communities, fungal communities, network analysis, microbial ecology 


\section{INTRODUCTION}

Plant microbiota are an important component that can influence essential plant functions positively or negatively. Better understanding of these host-microbe interactions has the potential to offer intervention tools to manipulate microbiota to enhance ecological services (Berg et al., 2020). Root-associated microbes have been shown to improve the systematically induced root exudation of metabolites process and affect root transcriptome and metabolome (Korenblum et al., 2020). Manipulation of plant microbiota thus trigger host biosynthetic and signaling pathways (Berg et al., 2016; Pascale et al., 2019) and brings up opportunities for plant fitness, improved nutrient usage efficiency, and higher crop yields while limiting chemical fertilizers and greenhouse gas emissions in a sustainable manner (Adesemoye et al., 2009; Berg, 2009; Singh and Trivedi, 2017; Ahmed and Hijri, 2021).

Cannabis sativa $\mathrm{L}$. is an emerging crop for the production of fiber, grain, and floral extracts that contribute to health and wellness, but is also widely cultivated indoors with a variety of growing substrates, artificial light, and temperature control. Some initial studies have explored ways to maintain yield (Backer et al., 2019; Saloner and Bernstein, 2020; Danziger and Bernstein, 2021) and encourage pathogen-free production (Taghinasab and Jabaji, 2020; Vujanovic et al., 2020), but few scientific studies on plant-associated microbiota have been conducted. Bacterial and fungal communities associated with the root, leaf, flower, and rhizosphere of the hemp cultivar C. sativa "Anka," have recently been studied in six locations in the Finger Lakes region of New York. This study identified a variety of microbes in each compartment, some of which are known to promote plant growth or contribute to plant resistance (Barnett et al., 2020). Barnett et al. (2020) identified candidate core microbiome members for each compartment sampled (Barnett et al., 2020). Recent studies showed that the C. sativa rhizosphere microbial community is determined by rhizosphere soil type and cultivar (Winston et al., 2014; Comeau et al., 2020). However, core microbiota provides essential associated microbial functions, as well as linkages between microbiota and their community structures (Zamioudis and Pieterse, 2011; Lebeis, 2014; Delgado-Baquerizo et al., 2018). Plant genotype, on the other hand, has a significant impact on associated microbiota (Sapkota et al., 2015). Hemp microbiota manipulation has been suggested as a potential to help with fiber processing (Law et al., 2020). Thus, significant consideration should be given to the microbiome of field-grown hemp, especially connecting yield and plant health. The development of effective microbiome-based techniques for improving yields and sustainable production of C. sativa is currently limited by the lack of understanding of associated microbes in multiple niches shaping the microbiome in relevant field settings.

Soil microbiomes provide important ecological services to natural and agricultural ecosystems. Given their potential for positively influencing agricultural productivity, understanding the role of these microbes could enhance our ability to exploit activities that promote efficient soil nutrient use to increase crop yield and quality, while reducing the environmental footprint of agriculture. Given the extraordinary number of microbes that exist in soil and their functional diversity, they could not be examined in depth prior to recent advances in high-throughput sequencing technologies and bioinformatics (Ahmed and Hijri, 2021), that allow to generate a huge amount of sequencing data whose analyses provide an exhaustive taxonomic profile, relative abundance as well as predition of functions. In this study, we used MiSeq amplicon sequencing targeting ribosomal DNA ( $\mathrm{rDNA}$ ) of bacterial $16 \mathrm{~S}$ and fungal ITS to uncover diversity of roots and rhizosphere soils associated with hemp grown in six different fields located in New York State (United States). We hypothesized that hemp is associated with a core microbiota, and that these taxa have distinct interaction patterns and are diverse in different biotopes. To test our hypothesis, we used high-throughput sequencing technologies to investigate the microbiome of a single clonally propagated cultivar of field grown hemp C. sativa "TJ's CBD" across six different field locations in New York State, where five plots were conventional and one plot was organic. We determined taxonomic abundance, indicator species, core microbiota, and interkingdom networking for identifying hub microbial taxa, which will help to target, select, and screen microbial taxa with high potential of biostimulant effect on hemp plants (Figure 1).

\section{MATERIALS AND METHODS}

\section{Experimental Design and Sampling}

The study was performed in six different fields in New York State, United States of America, from May 22 to October 11, 2019 (Table 1), in a randomized complete block design. All plants were started as rooted cuttings treated with Clonex rooting gel in the greenhouse using Lamberts LM-111 potting mix in 50-cell deep flats and transplanted to the field after 25-30 days. Each plot was planted with C. sativa "TJ's CBD," a clonal cultivar used for cannabidiol production. Only WOF was organic out of six fields. The hemp was planted in black plastic mulch, and weed control was performed by hoeing. Two tons of turkey manure per acre were applied to the WOF plot, and leek (Allium ampeloprasum L.) was the prior crop. All rhizosphere soil and root samples were collected in three replicates per field. The root system of each plant was collected using a shovel (approximately $15 \mathrm{~cm}$ depth). Fine roots were then cut and put them in a $50 \mathrm{~mL}$ tube. Rhizosphere soils were also collected on site and put in a separate container. First, we separated shoots from roots. Roots were gently shacked to remove bulk soil. The remaining soil attached to the roots was collected in Ziploc bags and it was considered as rhizosphere soil ( $\sim 500 \mathrm{mg}$; Floc'h et al., 2020$)$. This rhizosphere soil was visually examined to remove root fragments. Samples were collected in October 2019, and they were immediately transported the lab in an ice pack and stored at $-20^{\circ} \mathrm{C}$ until March 2020. These samples were thawed and used for DNA extractions.

\section{DNA Extraction, PCR, and Sequencing}

DNA extraction from roots and rhizosphere soils and PCR amplifications were carried out as described in Ahmed et al. (2021). Briefly, total 36 frozen hemp root and 


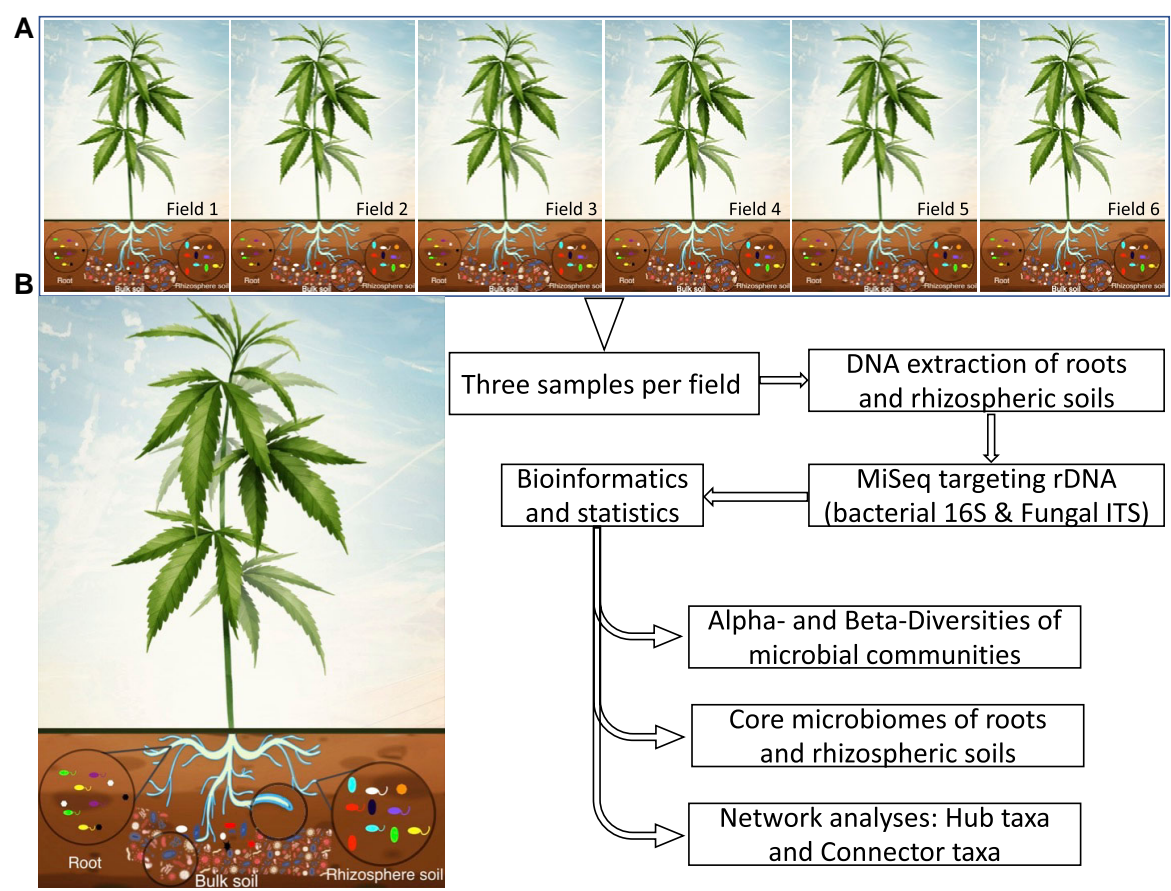

FIGURE 1 | Schematic illustration showing the experimental workflow of a clonal cultivar of hemp grown in six fields distributed at the State of New York (A). MiSeq sequencing and the DADA2 pipeline were used to determine community composition, and core microbes of roots and rhizosphere soils. Network analysis was performed to provide insight into the core microbial taxa and hub microbes. (B) Hypothetical core microbes of roots and rhizosphere soils selected from bulk soil microbiome. Rhizosphere soil is show by blue line surrounding roots.

TABLE 1 | Field locations, planting, and sampling dates.

\begin{tabular}{|c|c|c|c|c|}
\hline Field & Altitude & Location & Planting date & Sampling date \\
\hline Bluegrass Lane (BGL) & $\begin{array}{l}42.460647^{\circ} \mathrm{N} \\
76.463028 \mathrm{~W}\end{array}$ & 232 Bluegrass Lane, Ithaca, NY, Tompkins County & June 7, 2019 & Oct. 9, 2019 \\
\hline Hudson Valley Lab (HVL) & $\begin{array}{l}41.745647^{\circ} \mathrm{N} \\
73.967743^{\circ} \mathrm{W}\end{array}$ & 3357 Rt 9W, Highland NY 12528, Ulster County & June 3, 2019 & Oct. 11, 2019 \\
\hline $\begin{array}{l}\text { Long Island Horticultural Research } \\
\text { and Extension Center (LIH) }\end{array}$ & $\begin{array}{l}40.954766^{\circ} \mathrm{N} \\
72.710851^{\circ} \mathrm{W}\end{array}$ & 3059 Sound Ave., Riverhead, NY 11901, Suffolk County & May 22, 2019 & Oct. 1, 2019 \\
\hline McCarthy CBD Trial (MC) & $\begin{array}{l}42.895483^{\circ} \mathrm{N} \\
77.007547^{\circ} \mathrm{W}\end{array}$ & 2865 County Rd. 6, Geneva, NY, Ontario County & June 5, 2019 & Oct. 10, 2019 \\
\hline McCarthy Stress Trial (MSC) & $\begin{array}{l}42.896246^{\circ} \mathrm{N} \\
77.008210^{\circ} \mathrm{W}\end{array}$ & 2865 County Rd. 6, Geneva, NY, Ontario County & July 28, 2019 & Oct. 6, 2019 \\
\hline Wegman's Organic Farm (WOF) & $\begin{array}{l}42.776611^{\circ} \mathrm{N} \\
77.329667^{\circ} \mathrm{W}\end{array}$ & 4842 West Lake Rd, Canandaigua, NY Ontario County & July 9, 2019 & Oct. 8, 2019 \\
\hline
\end{tabular}

rhizosphere soil samples were considered for DNA extraction. First, we took $100 \mathrm{mg}$ roots were ground to make fine powder using pre-chilled mortars and pastels. Grounded roots that may contain endophytes were used for DNA extraction using the DNeasy Plant mini kit (Qiagen, Toronto, ON, Canada). DNeasy PowerSoil Pro kit (Qiagen, Toronto, ON, Canada) was used to extract DNA from $250 \mathrm{mg}$ of rhizosphere soil. Eluted DNA $(30 \mu \mathrm{L})$ was stored at $-20^{\circ} \mathrm{C}$. The NanoDrop ${ }^{\mathrm{TM}} 2000 / 2000 \mathrm{c}$ Spectrophotometer (ThermoFisher Scientific, Canada) was used to quantify the extracted DNAs, which were then visualized using a 1 percent agarose gel and the GelDoc System (BioRad, Montreal, QC, Canada). The bacterial 16S rDNA and fungal ITS regions were amplified by PCR. For the amplification of bacterial 16S rDNA, we used forward primer CS1_341 (5'-ACA CTGACGACATGGTTCTACACCTACGGGNGGCWGCAG-3') and reverse primer CS2_806R (5'-TACGGTAGCAGAGACT TGGTCTGACTACHVGGGTATCTAATCC- $3^{\prime}$ ), while fungal ITS region was amplified with CS1_ITS3_KYO2 (5'-ACACTG ACGACATGGTTCTACAGATGAAGAACGYAGYRAA- $3^{\prime}$ ) and CS2_ITS4 (5'-TACGGTAGCAGAGACTTGGTCTTCCTCCG CTTATTGATATGC-3'; Toju et al., 2012). In addition, 16S bacterial primers were used to target V3 and V4 regions of 16S rDNA while ITS primers targeted ITS2 region located between $5.8 \mathrm{~S}$ and $25 \mathrm{~S}$ genes of rRNA. The PCR reactions were performed using 1.5X Platinum ${ }^{\mathrm{TM}}$ Direct PCR Universal Master Mix (ThermoFisher, Montreal, QC, Canada), $0.25 \mu \mathrm{M}$ of each 
primer, 1.5X Platinum ${ }^{\mathrm{TM}}$ GC Enhancer, and $20 \mathrm{ng}$ of template DNA in a $25 \mu \mathrm{L}$ reaction volume. PCR reactions were run in an Eppendorf Mastercycler Pro S (Eppendorf, ON, Canada) with following cycling conditions: activation at $94^{\circ} \mathrm{C}$ for $2 \mathrm{~min}$, followed by 35 cycles of denaturation at $94^{\circ} \mathrm{C}$ for $15 \mathrm{~s}$, annealing at $60^{\circ} \mathrm{C}$ for $15 \mathrm{~s}$, extension at $68^{\circ} \mathrm{C}$ for $20 \mathrm{~s}$, a final extension at $68^{\circ} \mathrm{C}$ for $1 \mathrm{~min}$ with a hold at $10^{\circ} \mathrm{C}$. Negative PCR controls without DNA were included in each PCR run. PCR products were visualized on $1 \%$ agarose gel stained by GelRed on a GelDoc system (BioRad, Saint-Laurent, QC, Canada). Amplicons were sent to the Genome Quebec Innovation Centre for sequencing on an Illumina MiSeq sequencer (San Diego, CA, United States; Montreal, QC, Canada) using 2 X 300 bp pair-end reads which were demultiplexed on the instrument.

\section{Sequence Processing and Analysis of Data}

R4.0.2 (R Core Team, 2020) was used for all bioinformatics tasks, including raw sequencing read processing and graphical analysis. Detailed information on bioinformatics pipeline and data processing are available in the Supplementary Information (Methods 1). Briefly, DADA2 was used to generate the Amplicon Sequence Variants (ASV) table, and taxonomy was assigned to ASV using the reference datasets SILVA (the Silva Project's version 138) for $16 \mathrm{~S}$ rDNA (Quast et al., 2013) and UNITE (version 8.3) for ITS (Nilsson et al., 2018). Using $\mathrm{R}$ and dplyr v2.0.0 (Wickham and Wickham, 2020), the relative abundance of taxa was calculated. To characterize species diversity in the communities, we used Shannon and Simpson's index for both abundance and evenness of the species presented in the community. We used the "rrarefy" function of the vegan package v 2.5-6 to normalize the dataset before calculating diversity indices (Oksanen et al., 2020). The Shannon and Simpson diversity indices were calculated using the vegan $\mathrm{v}$ 2.5-6 on R. We calculated species evenness using Pielou's index $[J=H / \mathrm{In}$ $(S)$ where $H$ is Shannon diversity index and $S$ referred the total number of species in the dataset] using vegan package $v$ 2.5-6 on R. Principle Coordination Analysis (PCoA) were calculated using Bray-Curtis distance matrix of Hellinger transformed counts using the $\mathrm{R}$ package vegan $\mathrm{v}$ 2.5.6. After the calculation of alpha diversity indices by ANOVA, we performed Tukey's post-hoc tests to compare between hemp fields and sample types using package agricolae v1.3-3 (Peşteanu and Bostan, 2020) on R. Permutation-based multivariate analysis of variance (PERMANOVA; Anderson, 2001) was performed on samples considered for investigation of interaction effects of certain drivers on bacterial and fungal community with the function "Adonis" of the R package vegan v 2.5-6 using Hellingertransformed and permutations 999 (Oksanen et al., 2019). With metacoder $\mathrm{v}$ 0.3.4, we visualized taxonomic abundance at the order level (Poisot et al., 2017). According to the definition of "core plant microbiota" (Vandenkoornhuyse et al., 2015), we defined core microbiota which is made up of taxa that are present in $100 \%$ of samples, root or rhizosphere soil associated with the host in different fields. We used the package indicspecies v 1.7.9 (De Cáceres and Jansen, 2019) using Šidák correction for multiple comparison in the $\mathrm{R}$ package RVAideMemoire $\mathrm{v}$ 0.9-78 (Hervé and Hervé, 2020). The algorithm "glasso" of the SPIEC-EASI v 1.0.6 (Kurtz et al., 2015) was used to perform a cooccurrence network analysis and the results were then exported into Cytoscape v 3.8.0 for visualization (Shannon et al., 2003). Edges were described as co-occurrences or mutual exclusions of positive or negative inverse covariance values between nodes. Betweenness centrality and degree emphasize central nodes as well as provide information about network architecture. Betweenness centrality is defined as the ratio of the shortest path between all other nodes in the network involving the given node. A ratio of betweenness centrality and degree of connectivity more than $95 \%$ of network taxa may indicate community participation in multipartite co-occurrences, allowing us to define strongly interconnected taxa as hub-taxa. The Venn diagrams were generated using the "Calculate and draw Venn diagrams" tool available on VIB, University of Gent ${ }^{1}$.

\section{RESULTS}

\section{Sequencing and Bioinformatics}

We sampled root and rhizosphere soil of $C$. sativa (hemp) in six fields in New York: Bluegrass Lane (BGL), Hudson Valley Lab (HVL), Long Island Horticultural Research and Extension Center (LIH), McCarthy CBD Trial (MC), McCarthy Stress Trial (MSC), and Wegman's Organic Farm (WOF). Three plants per field, for a total of 18 plants, were sampled for root and rhizosphere soil. Illumina MiSeq produced a total of 11,617,362 pair-end raw reads $(5,639,337$ from bacteria and 5,978,025 from fungi). The number of reads per sample ranged from 41,561 to 98,764 for bacteria, and 34,891 to 106,235 for fungi. Using the DADA2 pipeline, 7160 bacterial and 3993 fungal ASVs were obtained. We also removed 26 ASVs from bacteria and 862 ASVs from fungi whose taxonomy belonged to mitochondria or chloroplast, leaving 7134 ASVs for bacteria and 3131 ASVs for fungi, respectively.

\section{Microbial Diversity Patterns in Field Grown Hemp: Compartment and Field-Dependent Effects}

The Shannon diversity index for bacteria is not as consistent as the Simpson index across all six hemp fields. The WOF field has the highest diversity mean in the Bacterial Shannon diversity index, whereas the BGL field has the lowest. However, the Simpson diversity index for all six fields is close to the maximum (Figure 2A). The Shannon diversity index was highly significant $(P=1.81 \mathrm{E}-05)$ on bacteria across fields (Supplementary Table 1). The BGL field was significant from HVL $(P<0.001)$, LIH $(P=0.02)$, MC $(P=0.008)$, MSC $(P=0.003)$, and WOF $(P=6.74 \mathrm{E}-06)$, and the LIH field was significant $(P=0.02)$ from WOF. The Pielou's evenness index did not varied much but BGL field had the lowest mean evenness index and there is a significant difference $(P=0.04)$

\footnotetext{
${ }^{1}$ http://bioinformatics.psb.ugent.be/webtools/Venn/
} 
A

Shannon

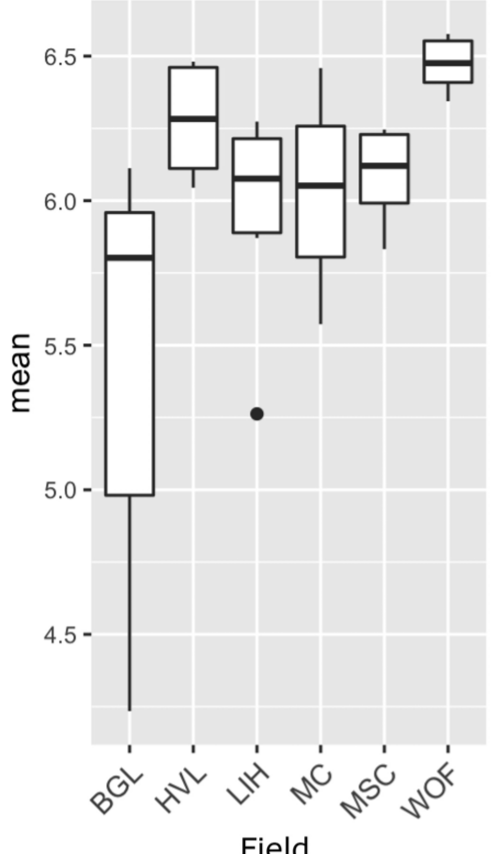

B

Shannon

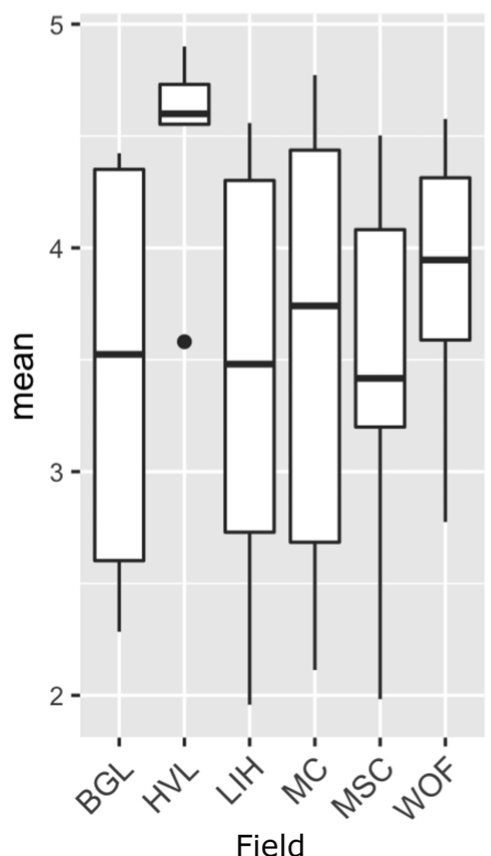

Simpson

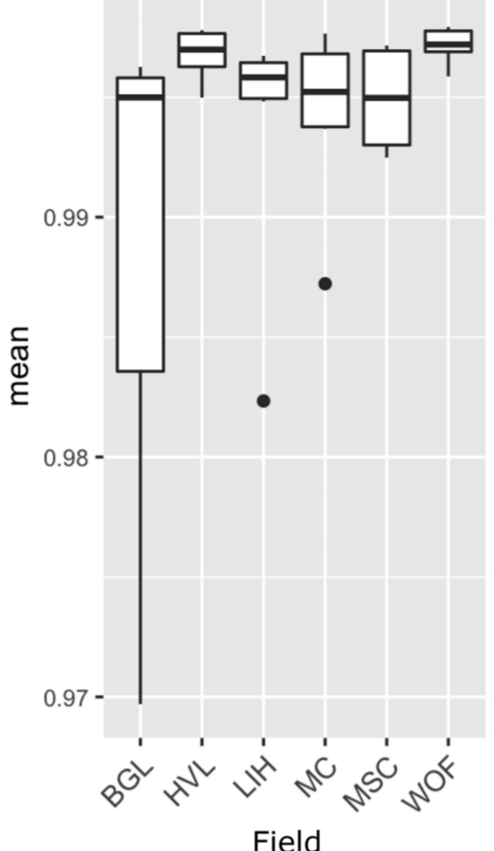

Simpson

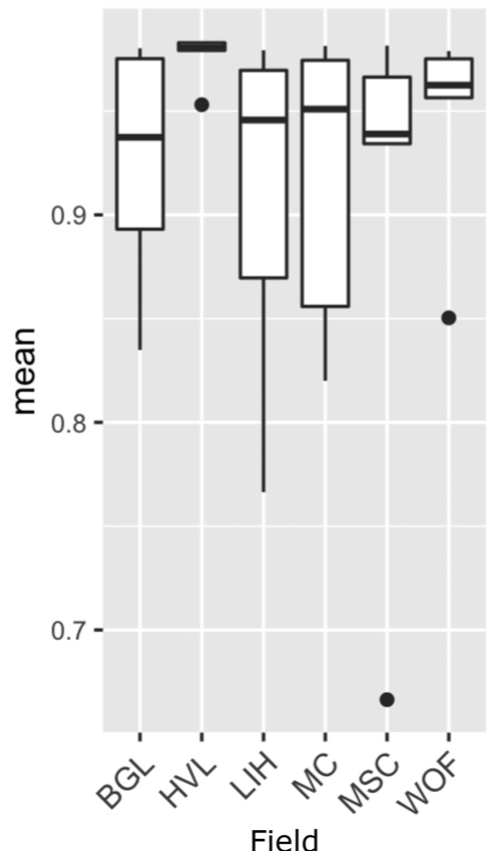

Pielou

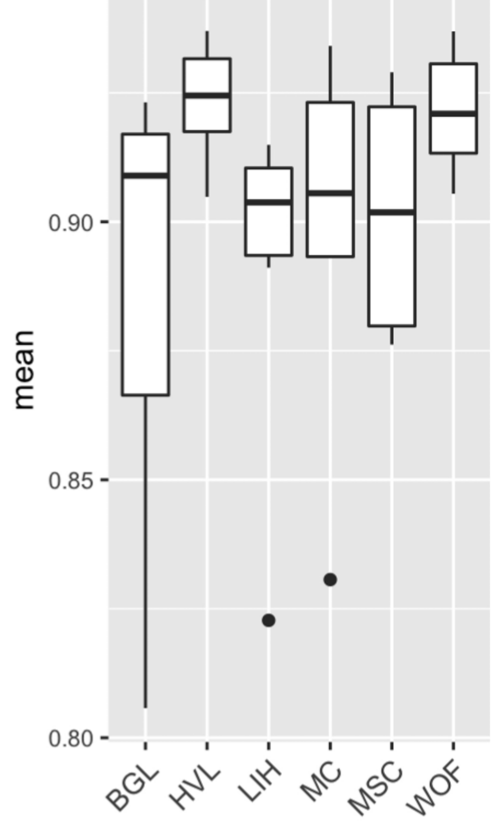

Field

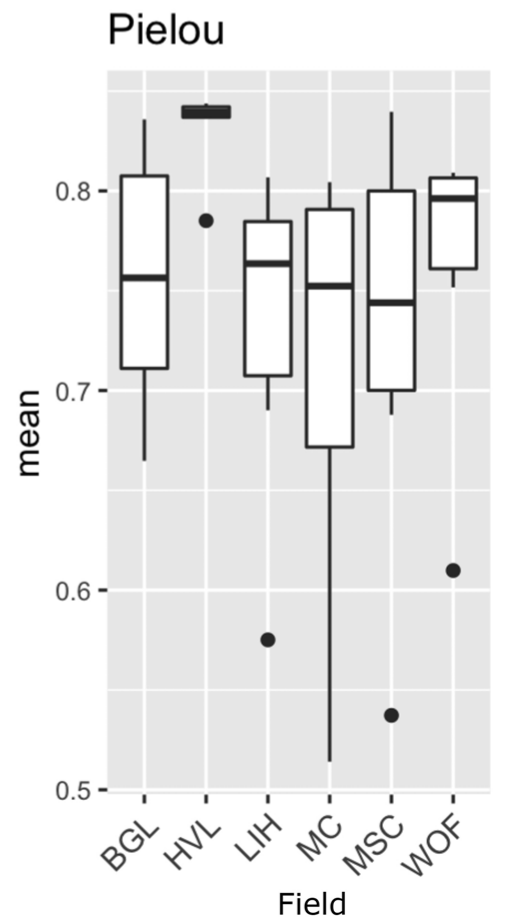

FIGURE 2 | Microbial species diversity and evenness in different hemp fields. The analysis of alpha diversity indices (Shannon and Simpson) and Pielou's evenness index of the bacterial and fungal communities: (A) bacterial microbiota; (B) fungal microbiota. Indices are shown according to fields. Field name: BGL, Bluegrass Lane; HVL, Hudson Valley Lab; LIH, Long Island Horticultural Research and Extension Center; MC, McCarthy CBD Trial; MSC, McCarthy Stress Trial; and WOF, Wegman's Organic Farm. Three replicates were used for the analysis of species diversity and evenness.

of species evenness in the bacterial communities (Figure 2A and Supplementary Table 1). For Fungi, the HVL field has the highest mean diversity according to the Shannon diversity index and the difference was statistically significant $(P=0.01)$. The Simpson diversity index is quite homogenous across fields (Figure 2B) and there is no significant difference between 
hemp fields (Supplementary Table 1). Although the mean of Pielou's evenness index is quite higher in HVL field, there is no statistically significant difference observed in the fungal communities across fields (Figure 2B). However, sample type (root or rhizosphere soil) had a significant effect on the bacterial (Shannon $P=1.12 \mathrm{E}-05$, Simpson $P=0.001$ ) and fungal diversity (Shannon $P=9.18 \mathrm{E}-08$, Simpson $P<0.001$ ). The Pielou's evenness index also showed significant difference for sample type in the bacterial (Pielou $P=2.00 \mathrm{E}-05$ ) and fungal communities (Pielou $P<001$; Supplementary Table 1). The diversity of bacteria in the WOF plot, which was the only organic field in the study, tend to be high for Shannon and Simpson indices, but the difference was not highly noticeable. Organic and conventional fields have similar microbial diversity and community structure. Bacterial communities clustered by biotopes according to the PCoA ordination (Figures $\mathbf{3 A}, \mathbf{B}$ ). Root associated bacteria in HVL and LIH formed strong clusters out of six hemp fields. In the rhizosphere soil, the bacterial community structure was clustered in three fields: LIH, MC, and MSC (Figure 3A). That said, fungal communities clustered differently than bacterial communities. For root biotopes, only MSC displayed a clear clustering pattern, while rhizosphere soil biotopes were clustered strongly in three fields: HVL, MSC, and WOF (Figure 3B). Using the PERMANOVA test, we observed that hemp fields revealed highly significant variations in both compartments in the bacterial (root: $R^{2}=0.611, P=0.001$, and rhizosphere soil: $R^{2}=0.807, P=0.001$ ) and fungal (root: $R^{2}=0.425$, $P=0.001$, and rhizosphere soil: $\left.R^{2}=0.485, P=0.001\right)$ community structure (Table 2).

\section{Differential Taxonomic Profiles in Hemp-Associated Microbial Community}

In the bacterial communities, we found 26 abundant phyla (Supplementary Table 2), with Planctobacteria being the most abundant phyla in both root (Figure 4A) and rhizosphere soil (Figure 4C). The 7,134 bacterial ASVs (BASV) were assigned to 108 orders (Supplementary Table 3). We chose

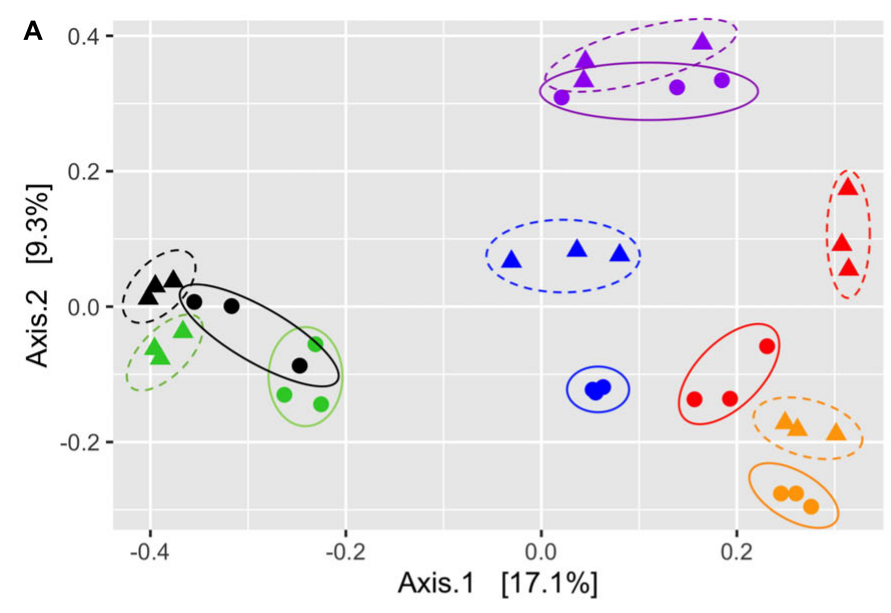

B

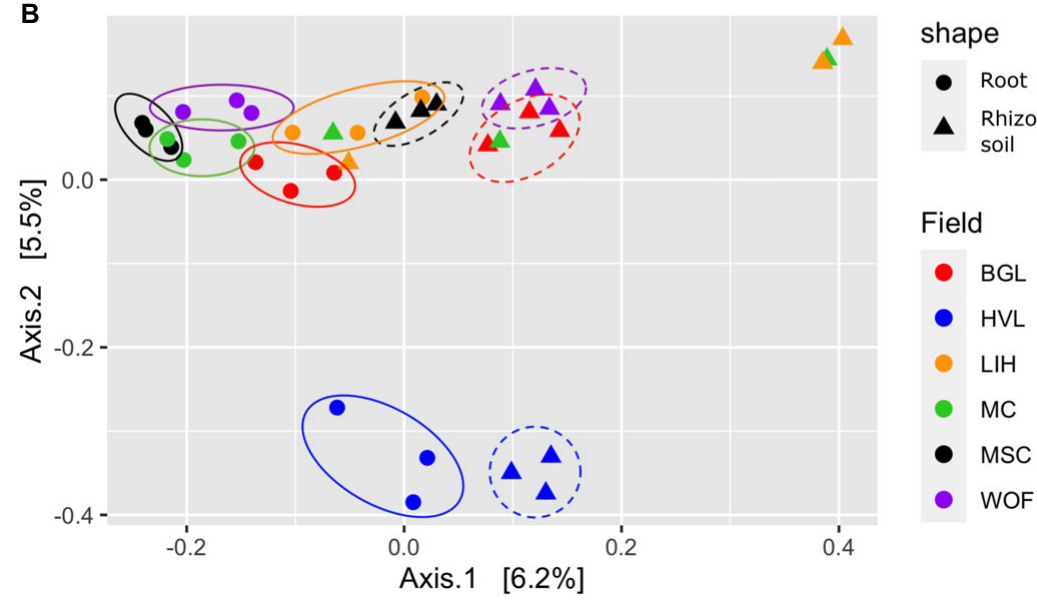

Axis. 1 [17.1\%]

$$
\text { (2) }
$$

shape

- Root

- Rhizosphere

Field

- BGL

- HVL

- LIH

- MC

- MSC

- WOF 
TABLE 2 | Effect of fields on the structure of the bacterial and fungal communities in root and rhizosphere soil according to PERMANOVA.

\begin{tabular}{lcccccc}
\hline Variable & Source & DF & SumOfSqs & $\boldsymbol{R}^{\mathbf{2}}$ & $\boldsymbol{F}$ & $\operatorname{Pr}(>\mathbf{F})$ \\
\hline (A) Bacteria & & & & & & \\
Roots & Field & 5 & 3.595 & 0.611 & 3.778 & $0.001^{* * *}$ \\
& Residual & 12 & 2.283 & 0.388 & & \\
& Total & 17 & 5.878 & 1.000 & & \\
Rhizosphere soil & Field & 5 & 3.676 & 0.807 & 10.069 & $0.001^{* * *}$ \\
& Residual & 12 & 0.876 & 0.192 & & \\
(B) Fungi & Total & 17 & 4.552 & 1.000 & & \\
Roots & & & & & & \\
& Field & 5 & 2.946 & 0.425 & 1.774 & $0.001^{* * *}$ \\
& Residual & 12 & 3.985 & 0.575 & & \\
Rhizosphere soil & Total & 17 & 5.878 & 1.000 & & \\
& Field & 5 & 2.785 & 0.485 & 2.257 & $0.001^{* * *}$ \\
& Residual & 12 & 2.962 & 0.515 & & \\
& Total & 17 & 5.748 & 1.000 & & \\
\hline
\end{tabular}

${ }^{* * *}$ means significance at $P=0.001$.

the top 10 orders based on their high relative abundance in both biotopes and eight of the 10 most abundant orders (Pirellulales, Gemmatales, Tepidisphaerales, Planctomycetales, Chthoniobacteriales, Isophaerales, Candidatus Kaiserbacteria, and Phycisphaerales) were dominant in both root and soil (Figures 4B,D). Both biotopes were dominated by Pirellulales (Figures 4B,D).

In the fungal dataset, we identified 12 phyla (Supplementary Table 4), with Ascomycota being the most abundant in both root (Figure 5A) and rhizosphere soil (Figure 5C). The 3,132 fungal ASVs were classified into 110 orders (Supplementary Table 5). The order Hypocreales dominated both biotopes followed by Agaricales, Sordariales, Cantharellales, Pleosporales, Eurotiales, Mortierellales, Chaetothyriales, Orbiliales, and Myrmecridiales (Figures 5B,D). Based on relative abundance, Hypocreales, Sordariales, Pleosporales, Glomererellales, Mortierellales, Eurotiales, Coniochaetales, Heliotales, Agaricales, and Pezizales were the top 10 fungal orders in the rhizosphere soil (Figure 5D). Both the root and rhizosphere soil fungal communities shared six (Hypocreales, Agaricales, Sordariales, Pleosporales, Eurotiales, and Mortierellales) of the top 10 orders (Figures 5B,D).

\section{Indicator Species and Core Microbiota Across Six Hemp Fields}

The indicator species analysis with Šidák correction for multiple comparisons accounted for on average 1 to $65 \%$ relative abundance of all root bacteria and 1 to $50 \%$ relative abundance of all rhizosphere soil bacteria detected in six different fields (Figure 6). However, fungal indicator species were not found in every field, for example, ASV7 (Fusarium equiseti) found in root in WOF (Figure 6C), in HVL and MSC one ASV was identified in the rhizosphere soil (Figure 6D). In root, WOF had the greatest number of bacterial indicator species (15), while in the rhizosphere soil, BGL had the most (30; Figure 6B). BGL had a maximum of six fungi in the rhizosphere soil (Figure 6D and Supplementary Table 6). Core microbiome composed of a small number of relatively abundant bacterial and fungal ASVs for each field. Here, we used the term "BASV" defining Bacterial ASV and "FASV" for fungal ASV. We found eight BASV as rhizosphere soil core microbiota, which were identified as four Planctobacteria (Gimesia maris, Pirellula sp. Lacipirellula limnantheis, and Gemmata sp.) and three of which were Unclassified Planctobacteria (Supplementary Figure 1A and Supplementary Table 7). Fusarium oxysporum, Gibellulopsis piscis, and Fusarium equiseti were identified as fungal core microbiota (Supplementary Figure $\mathbf{1 B}$ and Supplementary Table 8).

\section{Network Patterns of Microbial Community in Field Grown Hemp}

Co-occurrence network analysis of the hemp microbiota revealed complex co-occurrences among bacterial and fungal ASVs in the soil than in the root, of which 167 nodes and 257 edges in the root and 448 nodes and 2,164 edges in the rhizosphere soil. Based on the betweenness centrality score and node degree, eight BASV, BASV26 (Lacipirellula limnantheis), BASV33 (Unidentified Tepidisphaerales), BASV37 (Caulifigura coniformis), BASV57 (Unidentified Pirellulaceaes), BASV194 (Unidentified Tepidisphaerales), BASV285 (C. coniformis), BASV322 (Unidentified Planctomycetes), and BASV425 (C. coniformis) were identified as hub taxa in the root (Figure 7A and Supplementary Figure 2A). Four BASV, BASV37 (C. coniformis), BASV314 (Unclassified Tepidisphaerales), BASV636 (Unclassified Gemmatales), BASV1686 (Pirellula sp.), and two fungal ASVs, FASV46 (Metarhizium marquandii) and FASV79 (Nectria ramulariae), were identified as hub microbes in the rhizosphere soil microbiome network (Figure $\mathbf{7 B}$ and Supplementary Figure 2B). BASV37 (C. coniformis) was the only hub taxa found both in the root and rhizosphere soil (Figure 7 and Supplementary Figure 2). The network of each hub taxon and its connected ASVs is termed to as a module, and Modular hub taxa were ASV-centered within a module. We identified a meta co-occurrence pattern of 13 modules (Figure 8). We also identified taxa connecting different modules and considered as connector taxa, and the taxa connecting within modules and in general are considered as network hub taxa. Detailed taxonomic information of modular hub, connector and network hub can be found in the Table 3. Their co-occurrence pattern revealed: (i) root modular hub ASVs, BASV425, BASV285, BASV57, BASV33, and BASV26 are connected, and four connector ASVs, BASV406, BASV108, BASV27, and BASV1 established co-occurrences between rhizosphere soil modular hub (BASV636 and BASV1686) and root modular hub taxa; (ii) rhizosphere soil modular hub BASV636 and BASV314 are negatively co-occurred via BASV916; (iii) rhizosphere soil hub taxa FASV79 had negative co-occurrences with BASV314 and BASV1686; (iv) a connector taxon, BASV1090 had positive co-occurrence with rhizosphere soil modular hub BASV314 but negative co-occurrence with the modular hub FASV46; (v) FASV264 connected maximum four modular hub taxa (FASV46, FASV79, BASV37, and BASV314). BASV27 positively co-occurrence two rhizosphere soil modular hubs, BASV285 and BASV57, but negatively with a rhizosphere 


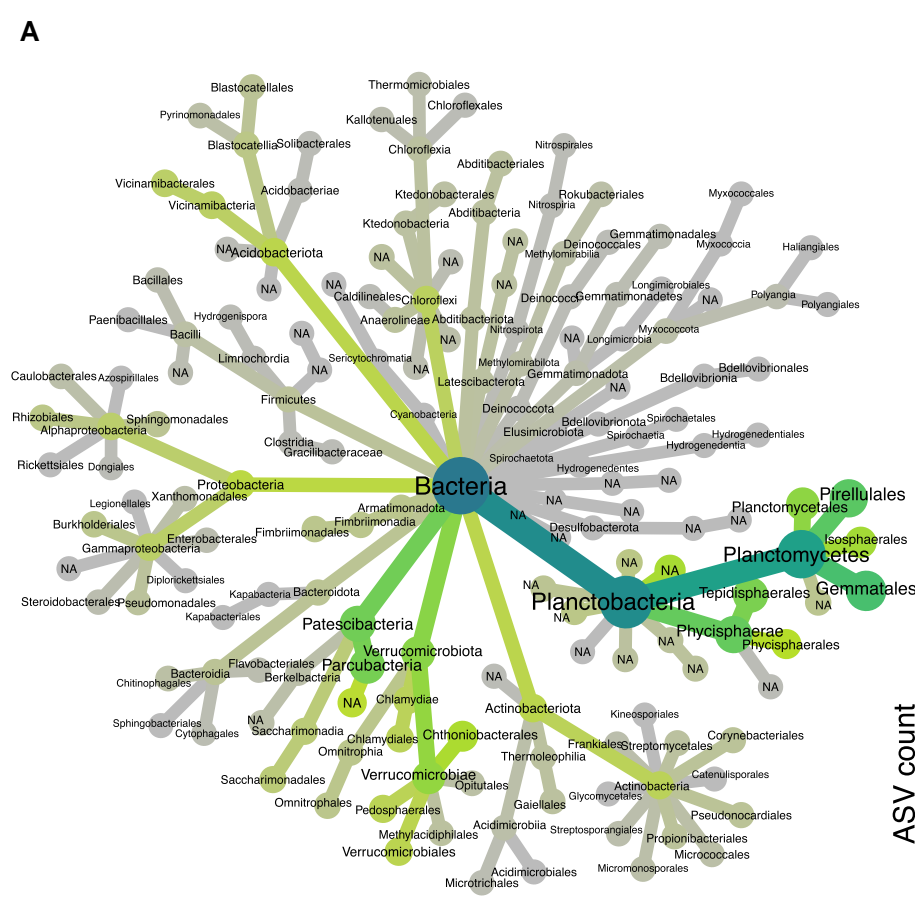

C

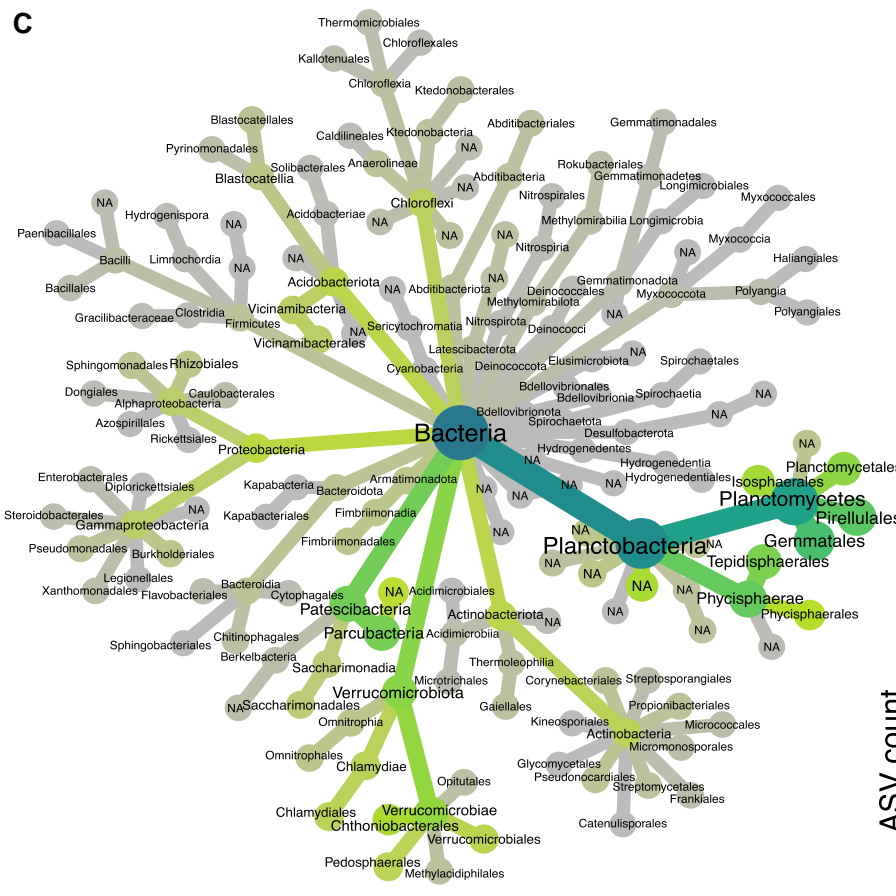

B

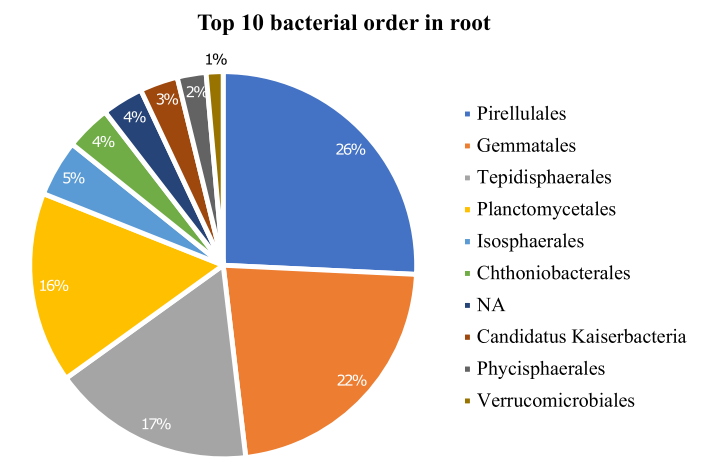

B

Nodes
D

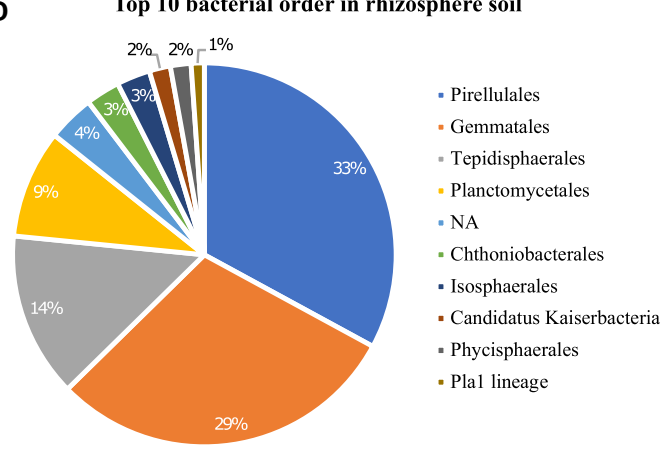

FIGURE 4 | Taxonomic hierarchy abundance at the order level for bacterial communities. (A) Taxonomic representation at order level in root; (B) relative abundance of top 10 bacterial orders in root. (C) Taxonomic representation at order level in rhizosphere soil (D) relative abundance of top 10 bacterial orders in rhizosphere soil. Three replicates were used for this study.

soil modular hub taxon, BASV1686. BASV57 established positive co-occurrences with a rhizosphere soil modular hub FASV46 and negative co-occurrences with two rhizosphere soil modular hubs, BASV314 and FASV79; (vi) FASV18 was positively connected with BASV37 but negatively with FASV46; and (vii) root modular hub BASV194 was not found in the co-occurrences network of network hub taxa. We identified eight bacterial and two fungal ASVs connecting all modular hub taxa to extend 


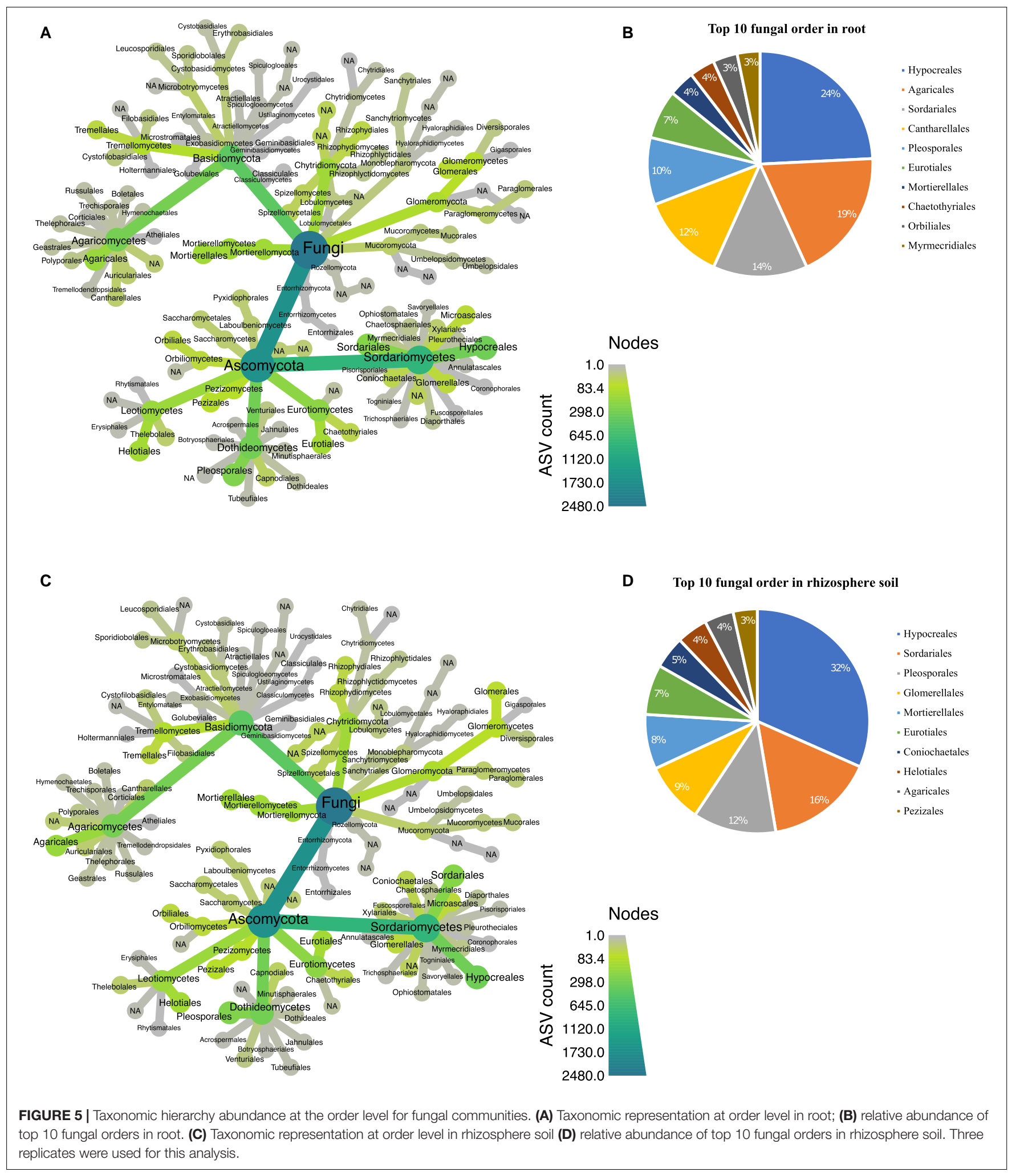

networks between the root and rhizosphere soil microbiota. Thus, these 10 connector ASVs and modular hubs, for a total of 24 ASVs have been chosen as network hub taxa (Figure 8 and Table 3). These 23 network hub taxa were identified as four bacteria and three fungi, and five ASVs were unidentified at the genus level were considered as network hub microbiota in the hemp microbiome network across six different field sites (Table 3). 
A

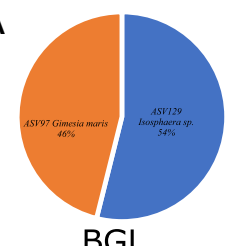

BGL

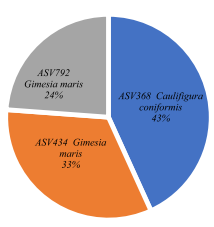

HVL

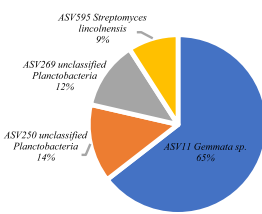

LIH

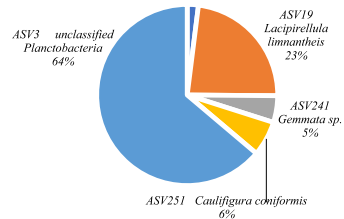

MC
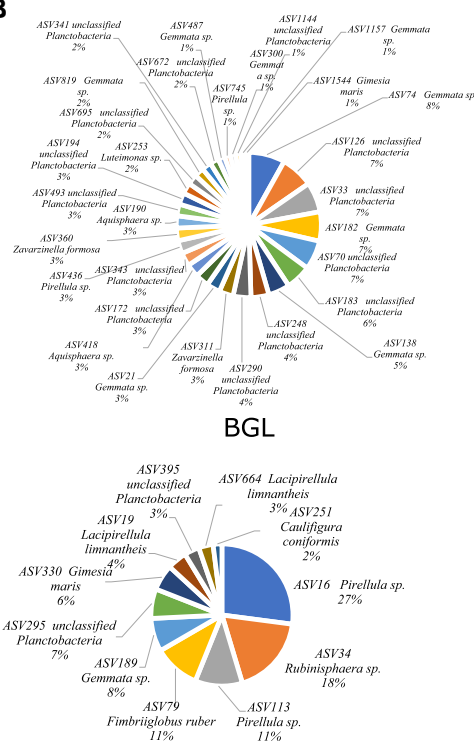

MC
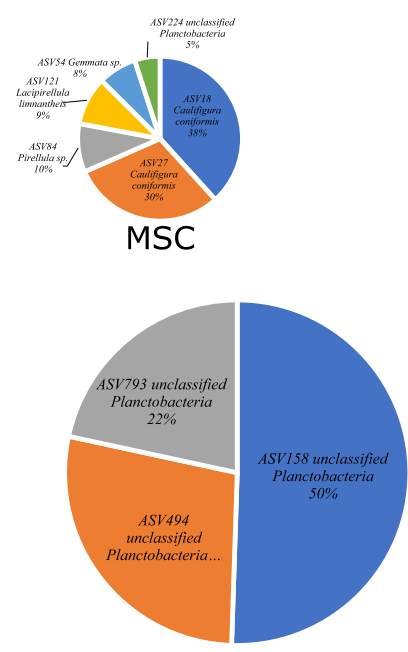

HVL

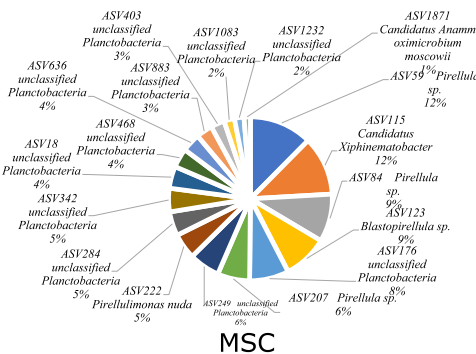

MSC

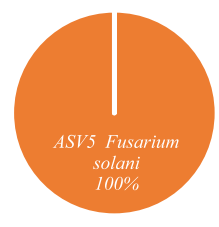

HVL
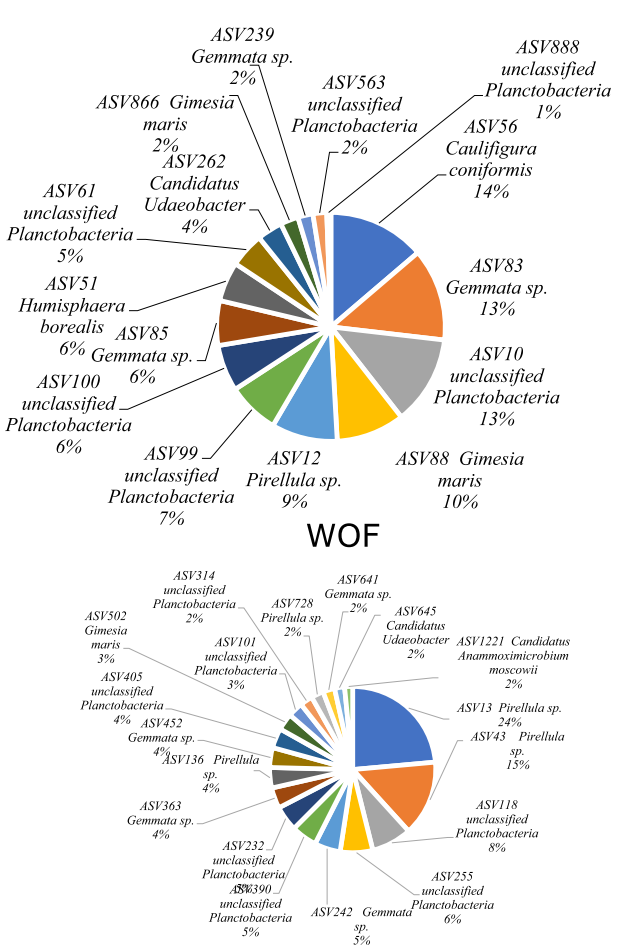

LIH

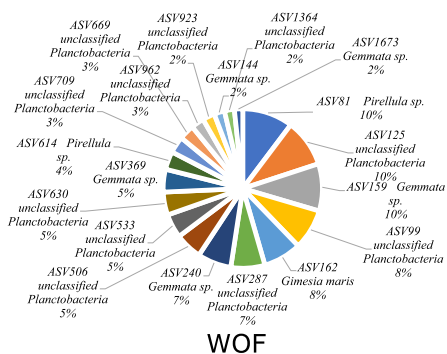

C

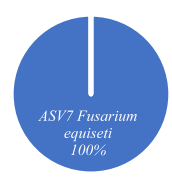

WOF

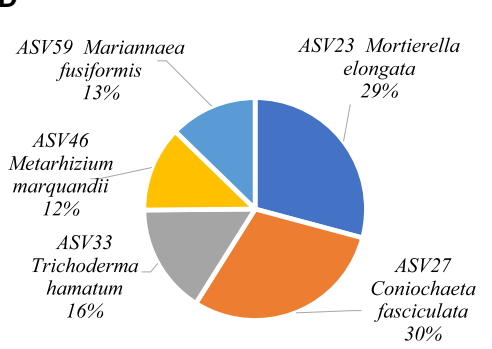

BGL
MSC
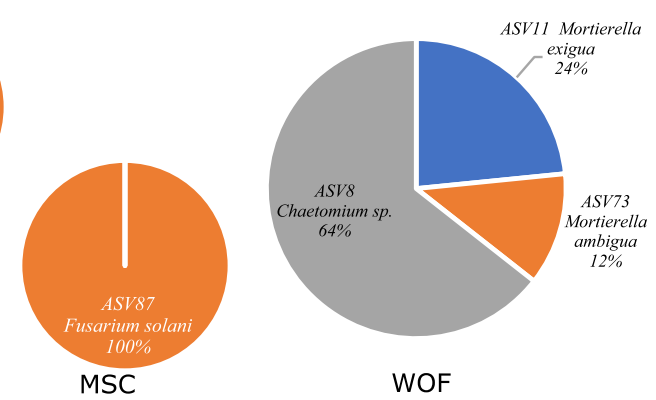

FIGURE 6 | Indicator species of hemp microbiome. The most representative species of hemp microbiome were identified through indicator species analysis in six different field locations. Bacterial indicator species in root (A) and in rhizosphere soil (B); and fungal indicator species in root (C); and in rhizosphere soil (D). Fungal indicator species in root identified only in WOF filed. Three replicates were used for analyzing indicator species in each field.

\section{DISCUSSION}

We studied the microbial communities associated with a single hemp cultivar growing in six field locations in New York State and different agricultural practices. Although the number of samples per field was low which may be a factor that influence statistical analyses, we therefore focus on comparisons of microbial communities in biotopes, the core, and the network across all samples. In hemp root and soil, we showed the diversity of bacterial and fungal communities, taxonomic abundance and interkingdom interactions. Planctobacteria and Ascomycota dominated bacterial and fungal communities in hemp microbiota 


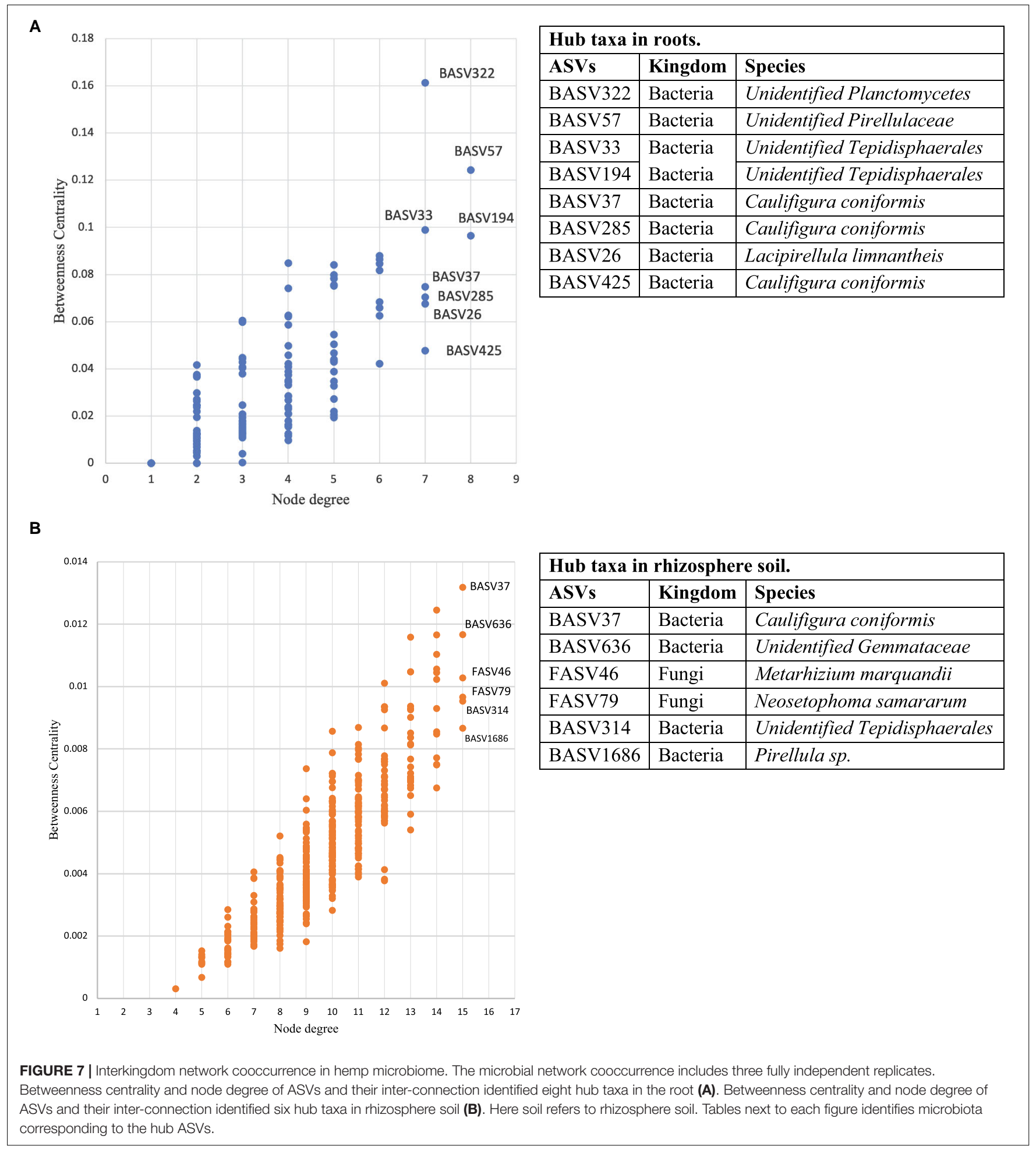

in six fields. Eight BASV and three fungal ASVs dominated the core microbiota. We discovered 24 ASVs as network hub taxa that are establishing microbial network associated with C. sativa TJ's CBD.

The alpha diversity of the microbial communities was greatly affected by compartment, but we found that community structure varied significantly among different hemp field sites. The community structure observed in this study resembled patterns found previously in hemp (C. sativa "Anka") growing in six fields in New York's Finger Lakes region (Barnett et al., 2020). Some of the main contributors to the taxa abundance in hemp in this study was Planctobacteria which was highly 


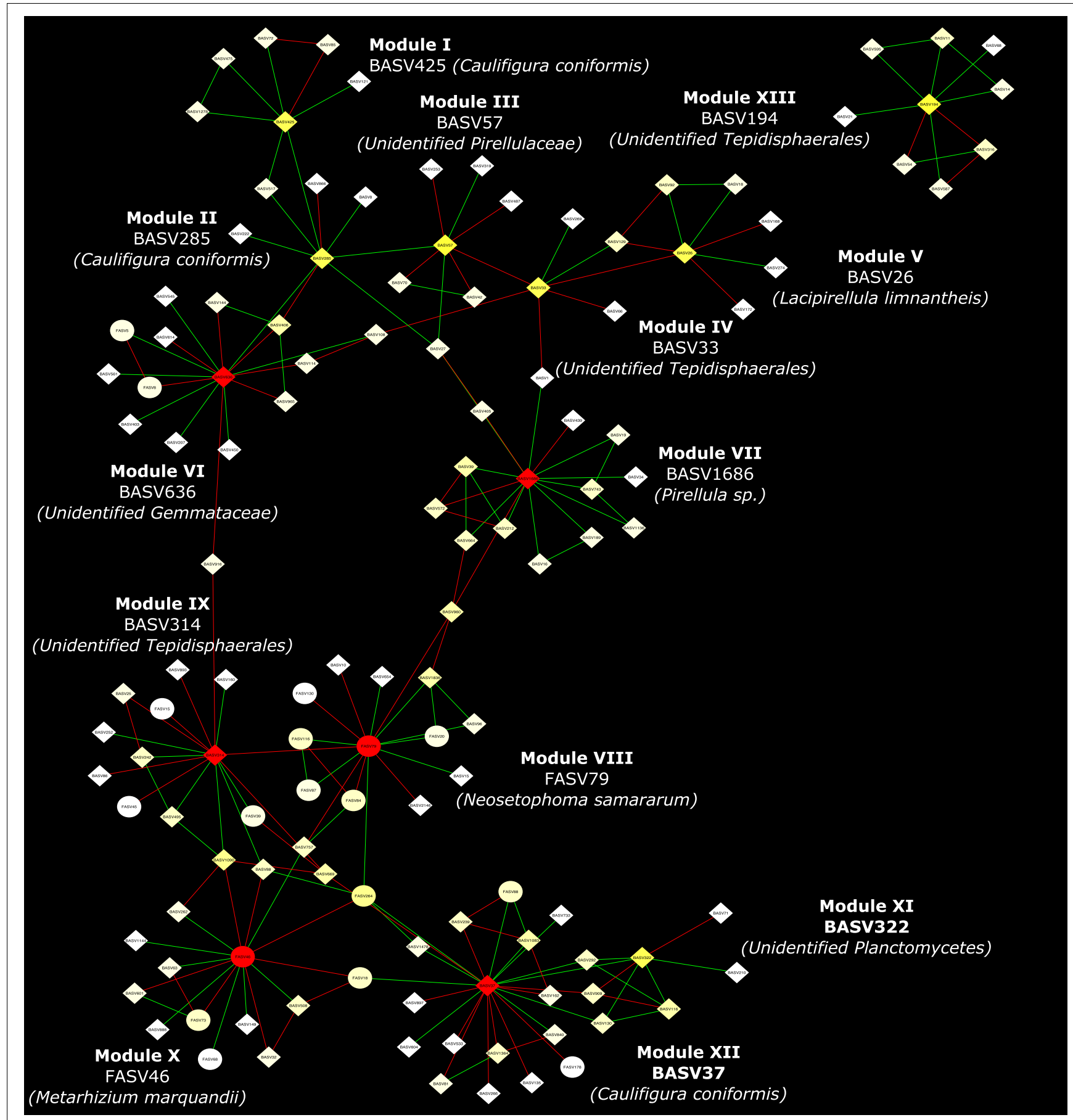

FIGURE 8 | Network hub taxa identified across six hemp fields in New York. Interkingdom interactions of hub taxa in the root and rhizosphere soil was analyzed using three replicates. The co-association of each hub taxa and its co-associated ASVs is denoted as a Module and ASV connecting between Modules considered as connector taxa. Hub taxa of the module and connector taxa combinedly considered as Network hub taxa. Here soil refers to rhizosphere soil.

enriched in root and rhizosphere soil biotopes, showed similar trends in the root and rhizosphere soil in other crops, such as soybean (Ahmed et al., 2021), soybean straw returns (Miao et al., 2020), and wild beet (Zachow et al., 2014). Planctomycetes (Planctobacteria) were observed in the highly abundant ( $>1 \%$ of the community) bacteria in multiple compartment (root, rhizosphere soil, bulk soil, flowers, and leaves) in field-grown hemp (Barnett et al., 2020). In support of Barnett et al. (2020) report of hemp associated fungi, we identified four of the top 10 fungal orders that were proven to have a greater association of those microbes with hemp, providing evidence that those fungal ASV s has an important impact in field-grown hemp in New York. 
TABLE 3 | Network hub taxa identified in six hemp fields.

\begin{tabular}{|c|c|c|c|c|c|c|c|}
\hline ASV_ID & Kingdom & Phylum & Class & Order & Family & Genus & Species \\
\hline BASV57 & Bacteria & Planctobacteria & Planctomycetes & Pirellulales & Pirellulaceae & $\begin{array}{l}\text { Unidentified } \\
\text { Pirellulaceae }\end{array}$ & $\begin{array}{l}\text { Unidentified } \\
\text { Pirellulaceae }\end{array}$ \\
\hline BASV194 & Bacteria & Planctobacteria & Phycisphaerae & Tepidisphaerales & WD2101 soil group & $\begin{array}{l}\text { Unidentified } \\
\text { Tepidisphaerales }\end{array}$ & $\begin{array}{l}\text { Unidentified } \\
\text { Tepidisphaerales }\end{array}$ \\
\hline BASV322 & Bacteria & Planctobacteria & Planctomycetes & Planctomycetales & Rubinisphaeraceae & $\begin{array}{l}\text { Unidentified } \\
\text { Planctomycetes }\end{array}$ & $\begin{array}{l}\text { Unidentified } \\
\text { Planctomycetes }\end{array}$ \\
\hline BASV33 & Bacteria & Planctobacteria & Phycisphaerae & Tepidisphaerales & WD2101 soil group & $\begin{array}{l}\text { Unidentified } \\
\text { Tepidisphaerales }\end{array}$ & $\begin{array}{l}\text { Unidentified } \\
\text { Tepidisphaerales }\end{array}$ \\
\hline BASV37 & Bacteria & Planctobacteria & Planctomycetes & Planctomycetales & Rubinisphaeraceae & Caulifigura & Caulifigura coniformis \\
\hline BASV285 & Bacteria & Planctobacteria & Planctomycetes & Planctomycetales & Rubinisphaeraceae & Caulifigura & Caulifigura coniformis \\
\hline BASV26 & Bacteria & Planctobacteria & Planctomycetes & Pirellulales & Lacipirellulaceae & Lacipirellula & Lacipirellula limnantheis \\
\hline BASV425 & Bacteria & Planctobacteria & Planctomycetes & Planctomycetales & Rubinisphaeraceae & Caulifigura & Caulifigura coniformis \\
\hline BASV636 & Bacteria & Planctobacteria & Planctomycetes & Gemmatales & Gemmataceae & $\begin{array}{l}\text { Unidentified } \\
\text { Gemmataceae }\end{array}$ & $\begin{array}{l}\text { Unidentified } \\
\text { Gemmataceae }\end{array}$ \\
\hline FASV46 & Fungi & Ascomycota & Sordariomycetes & Hypocreales & Clavicipitaceae & Metarhizium & $\begin{array}{l}\text { Metarhizium } \\
\text { marquandii }\end{array}$ \\
\hline FASV79 & Fungi & Ascomycota & Dothideomycetes & Pleosporales & Phaeosphaeriaceae & Neosetophoma & $\begin{array}{l}\text { Neosetophoma } \\
\text { samararum }\end{array}$ \\
\hline BASV314 & Bacteria & Planctobacteria & Phycisphaerae & Tepidisphaerales & WD2101 soil group & $\begin{array}{l}\text { Unidentified } \\
\text { Tepidisphaerales }\end{array}$ & $\begin{array}{l}\text { Unidentified } \\
\text { Tepidisphaerales }\end{array}$ \\
\hline BASV1686 & Bacteria & Planctobacteria & Planctomycetes & Pirellulales & Pirellulaceae & Pirellula & Pirellula sp. \\
\hline BASV406 & Bacteria & Planctobacteria & Planctomycetes & Gemmatales & Gemmataceae & Gemmata & Gemmata sp. \\
\hline BASV108 & Bacteria & Planctobacteria & Planctomycetes & Pirellulales & Pirellulaceae & $\begin{array}{l}\text { unidentified } \\
\text { Pirellulaceae }\end{array}$ & $\begin{array}{l}\text { unidentified } \\
\text { Pirellulaceae }\end{array}$ \\
\hline BASV1 & Bacteria & Planctobacteria & Planctomycetes & Pirellulales & Lacipirellulaceae & Lacipirellula & Lacipirellula limnantheis \\
\hline BASV916 & Bacteria & Planctobacteria & Planctomycetes & Gemmatales & Gemmataceae & $\begin{array}{l}\text { Unidentified } \\
\text { Gemmataceae }\end{array}$ & $\begin{array}{l}\text { Unidentified } \\
\text { Gemmataceae }\end{array}$ \\
\hline BASV960 & Bacteria & Planctobacteria & Planctomycetes & Pirellulales & Pirellulaceae & Pirellula & Pirellula sp. \\
\hline BASV1090 & Bacteria & Planctobacteria & Phycisphaerae & Pla1 lineage & NA & $\begin{array}{l}\text { Unidentified } \\
\text { Phycisphaerae }\end{array}$ & $\begin{array}{l}\text { unidentified } \\
\text { Phycisphaerae }\end{array}$ \\
\hline BASV27 & Bacteria & Planctobacteria & Planctomycetes & Planctomycetales & Rubinisphaeraceae & Caulifigura & Caulifigura coniformis \\
\hline BASV757 & Bacteria & Planctobacteria & Planctomycetes & Gemmatales & Gemmataceae & $\begin{array}{l}\text { Unidentified } \\
\text { Gemmataceae }\end{array}$ & $\begin{array}{l}\text { Unidentified } \\
\text { Gemmataceae }\end{array}$ \\
\hline FASV264 & Fungi & Ascomycota & Sordariomycetes & Hypocreales & Clavicipitaceae & Metarhizium & $\begin{array}{l}\text { Metarhizium } \\
\text { marquandii }\end{array}$ \\
\hline FASV18 & Fungi & Ascomycota & Sordariomycetes & Hypocreales & Nectriaceae & Fusarium & Fusarium oxysporum \\
\hline
\end{tabular}

For root and rhizosphere soil communities, the influence of field site was strongest, implying that several factors including soil chemistry were likely to be driving this field specific variance. Our study comprised six different field locations, each with unique soil features that could influence hemp-associated microbiota. Overall, our data indicated that while local environment factors may influence hemp microbiota, certain bacteria and fungi were abundant in hemp irrespective of fields location.

To learn more about microbial community patterns across fields and hemp-microbe interactions, we identified indicator species and the core microbiome. The indicator species analysis identifying different bacteria and fungi and their ties to field sites allows in predicting their presence and abundance of those taxa. In comparing to other fields, the rhizosphere fungal communities in LIH and MC are scattered. In LIH and MC, we found no fungal indicator species in the root and rhizosphere soil, which could explain why the LIH and MC fungal communities in our PCoA were so dispersed (Figure 3B). We found Streptomyces lincolnensis as an indicator species associated with hemp roots in LIH field. However, Streptomyces sp. has been reported as a potential biocontrol agent of plant pathogens as well as of plant growth enhancer (Figueiredo et al., 2010; de Jesus Sousa and Olivares, 2016; Vurukonda et al., 2018). Beneficial fungal species, Trichoderma hamatum and Metarhizium marquandii, were identified as fungal indicator species in the rhizosphere soil of BGL field. It has been proven that T. hamatum has biocontrol activity and potential to increase plant biomass extensively in agriculture practices (Harman et al., 2004; Studholme et al., 2013), such as it promotes lettuce growth in low $\mathrm{pH}$ and nutrient poor soil, and protect against pre-emergence disease caused by Rhizoctonia solani and Sclerotinia sclerotiorum (Ryder et al., 2012). Recently, Metarhizium marquandii was selected from an in vitro screening with another fungal species and observed in solubilizing phosphorus, producing indoleacetic acid and contributing to plant growth in soybean, maize, and bean (Baron et al., 2020).

No core microbiota was identified in hemp root, and a major portion of the bacterial core $(37 \%)$ was unclassified 
at the genus level, indicating that the core hemp microbiota comprises bacteria that have yet to be isolated or documented. This resulted in a list of microbes that should now be prioritized for directed isolation or genome binning using sequencing to further understand their potentials in hemp. We observed very little evidence of core microbiota. We identified a limited list of microbes as core microbiota (four bacteria and three fungi), which is similar to recent field-grown hemp study, who identified five bacterial and fungal core taxa in the bulk soil (Barnett et al., 2020). Fusarium solani identified as soil indicator species in HVL and MSC field sites. Fusarium oxysporium has been identified as a core fungal microbiota. Fusarium are documented for its devastating effects such as causing root and crow rot of C. sativa (Punja, 2021). But the presence of Fusarium as a core microbiota in bulk soil of field-grown hemp (Barnett et al., 2020), not only supports its common occurrence but also opens the possibility of studying the interactions of host-dependent pathogens to truly comprehend soil health of hemp fields. Fusarium spp. could be combined with other biocontrol agents to enhance hemp protection against pathogen attacks (Kaur et al., 2011). Beneficial bacteria may colonize Fusarium species, which could explain the positive correlations on the one hand and the hemp benefits on the other, which have been observed in canola (Lay et al., 2018), non-pathogenic F. oxysporum isolate Fo47 discovered from Fusarium wilt suppressive soils in France, has been intensively studied for the management of Fusarium wilt disease in a variety of vegetable and flower crops (Alabouvette et al., 1998). However, we did not investigate Fusarium's non-pathogenic activity in hemp. Therefore, future studies on functional activities, including biocontrol agents is needed. Our findings illustrate a list of prospective candidate microbes for further research into hemp-specific interactions and how their activity relates to hemp performance.

Plant microbial networks are complicated, and each contributing taxon performs specialized roles that are critical to the ecosystem's functionality, as a result, contribute to plant health (Zhou et al., 2010; Huang et al., 2020). Thus, it is essential to study microbial co-occurrences in the plant microbiome and how they impact plant health. We identified 24 ASVs as network hub taxa that maintain microbial community structure in root and rhizosphere soil. Since the hub microbes are crucial to plant health (Philippot et al., 2013; Agler et al., 2016), they could be ideal targets for new management practices intended to boost crop production. Microbial hub taxa can also link factors controlling the dynamics of plant associated microbiome and more effectively stabilizing selected microbiota according to plant genotype (Agler et al., 2016). As a result, their identity as well as functional role should be clarified. Addressing the microbial co-occurrences with hemp can enhance crop health and productivity by manipulating grow strategies. Given the fact that study into the hemp microbiome is still in its preliminary phase, data suggest that cultivar and soil type dependent selection are the primary factors in determining microbial community composition (Garbeva et al., 2004; Berg and Smalla, 2009; Taghinasab and Jabaji, 2020). We detected Chaetomium globosum as an indicator species in the rhizosphere soil of the WOF field (Supplementary Table 6), which confirms the previous reports of hemp endophytes (Kusari et al., 2013). Most of the microbiome studies in C. sativa have focused on high THC cultivars (McKernan et al., 2016; Comeau et al., 2020) as well as identifying endophytes (Gautam et al., 2013; Kusari et al., 2013; Scott et al., 2018; Taghinasab and Jabaji, 2020). However, there has been little progress in understanding of endophytic bacteria and fungi in various organs affects growth and biocontrol potentiality (Pagnani et al., 2018; Taghinasab and Jabaji, 2020) but the underlying mechanism is still unexplained. It's been proposed that bacteria isolated from one plant species might improve plant biomass and respond to stress in another, implying that beneficial microbes could enhance hemp production. Our results suggest that searching for field sites reliant microbial community assemblages can enhance hemp biomass, biocontrol pathogens, and secondary metabolites production, as previously explained (Taghinasab and Jabaji, 2020). The discovery of core and hub microbial members in field-grown hemp in New York state not only verifies the importance of common microbes in screening and assemblage of hemp microbiota (Barnett et al., 2020), but also opens up new prospects in studying whether there are any hemp-associated bacteria or fungus that could be used to produce biocontrol agents.

Plant microbiomes have huge potential to increase yield in a sustainable way to meet rising global food and biofuel demands. Substantial investigations are necessary to correctly assess the roles of microbial interactions with hemp in modern agricultural systems with the aim of integrating beneficial microbiomes into crop yields. By studying microbial diversity, community structure and interkingdom interactions between bacteria and fungi in hemp roots and rhizosphere soil in natural fields, as well as defining core and hub microbiota for further inquiry and manipulations, our study contributed to addressing key information gaps for hemp cultivar-byenvironment-microbiota interactions. Understanding the role of microbiome in distinct plant compartments and different developmental stages, as well as their role on the quality and quantity of yield and secondary metabolites production has been given importance (Barnett et al., 2020; Dastogeer et al., 2020; Taghinasab and Jabaji, 2020). Our study based on the microbiota at maturity stage; nevertheless, we may have missed some microbial members during other developmental stages. However, our findings endorse the idea that some bacterial and fungal taxa were shared by the clonal hemp cultivar "TJ's CBD" grown across six different field locations, and that such taxa seemed to be strongly associated with hemp regardless of location. This study could be effective in adding more understanding into the role of hemp-associated bacteria and fungus throughout plant development by merging microbiome and transcriptomics data.

\section{DATA AVAILABILITY STATEMENT}

The datasets presented in this study can be found in online repositories. The names of the repository/repositories and accession number(s) can be found below: all sequences 
are accessible in NCBI SRA database under the accession number PRJNA740043.

\section{AUTHOR CONTRIBUTIONS}

BA: conceptualization, experimental design, processed data, and wrote the manuscript. LS: conceptualization, experimental design, sample collection, supervision, and manuscript editing. $\mathrm{MH}$ : concepts, design, supervision, and contribution to the manuscript writing. All authors contributed to the article and approved the submitted version.

\section{FUNDING}

This study was supported by The Fonds de Recherche du Québec - Nature et Technologies (FRQNT) B3X fellowship and Mitacs Globalink to BA, Natural Sciences and Engineering Research Council (NSERC) Discovery grant to $\mathrm{MH}$ (Grant number: RGPIN-2018-04178) which are

\section{REFERENCES}

Adesemoye, A. O., Torbert, H. A., and Kloepper, J. W. (2009). Plant growthpromoting rhizobacteria allow reduced application rates of chemical fertilizers. Microb. Ecol. 58, 921-929. doi: 10.1007/s00248-009-9531-y

Agler, M. T., Ruhe, J., Kroll, S., Morhenn, C., Kim, S.-T., Weigel, D., et al. (2016). Microbial hub taxa link host and abiotic factors to plant microbiome variation. PLoS Biol. 14:e1002352. doi: 10.1371/journal.pbio.1002352

Ahmed, B., and Hijri, M. (2021). Potential impacts of soil microbiota manipulation on secondary metabolites production in cannabis. J. Can. Res. 3:25. doi: 10 . 1186/s42238-021-00082-0

Ahmed, B., Floc'h, J.-B., Lahrach, Z., and Hijri, M. (2021). Phytate and microbial suspension amendments increased soybean growth and shifted microbial community structure. Microorganisms 9:1803. doi: 10.3390/ microorganisms 9091803

Alabouvette, C., Schippers, B., Lemanceau, P., and Bakker, P. (1998). "Biological control of fusarium wilts: toward development of commercial products," in Plant-Microbe Interactions and Biological Control, eds G. J. Boland and L. D. Kuykendall (New York: Marcel Deckker), 15-38.

Anderson, M. J. (2001). A new method for non-parametric multivariate analysis of variance. Austral. Ecol. 26, 32-46. doi: 10.1111/j.1442-9993.2001.010 70.pp.x

Backer, R., Schwinghamer, T., Rosenbaum, P., Mccarty, V., Eichhorn Bilodeau, S., Lyu, D., et al. (2019). Closing the yield gap for cannabis: a meta-analysis of factors determining cannabis yield. Front. Plant Sci. 10:495. doi: 10.3389/fpls. 2019.00495

Barnett, S. E., Cala, A. R., Hansen, J. L., Crawford, J., Viands, D. R., Smart, L. B., et al. (2020). Evaluating the microbiome of hemp. Phytob. J. 4, 351-363.

Baron, N. C., De Souza Pollo, A., and Rigobelo, E. C. (2020). Purpureocillium lilacinum and Metarhizium marquandii as plant growth-promoting fungi. PeerJ 8:e9005. doi: $10.7717 /$ peerj.9005

Berg, G. (2009). Plant-microbe interactions promoting plant growth and health: perspectives for controlled use of microorganisms in agriculture. Appl. Microbiol. Biotechnol. 84, 11-18. doi: 10.1007/s00253-009-2092-7

Berg, G., and Smalla, K. (2009). Plant species and soil type cooperatively shape the structure and function of microbial communities in the rhizosphere. FEMS Microbiol. Ecol. 68, 1-13.

Berg, G., Rybakova, D., Fischer, D., Cernava, T., Vergès, M.-C. C., Charles, T., et al. (2020). Microbiome definition re-visited: old concepts and new challenges. Microbiome 8:103.

Berg, G., Rybakova, D., Grube, M., and Köberl, M. (2016). The plant microbiome explored: implications for experimental botany. J. Exp. Bot. 67, 995-1002. doi: 10.1093/jxb/erv466 gratefully acknowledged. This study was partially funded by New York State Department of Agriculture and Markets through grants (AC477 and AC483) from Empire State Development.

\section{ACKNOWLEDGMENTS}

We thank Mark Sisson, Theresa Rusinek, George Stack, Rebecca Wilk, Savanna Shelnutt, and Deanna Gentner for technical assistance and sample collection, and Jean-Baptiste Floc'h and Jean Legeay for statistical analysis assistance. We are grateful to Stem Holdings Agri for providing “TJ's CBD” for research purposes. We also thank Mr. Said Farer for assistance in the design of Figure 1.

\section{SUPPLEMENTARY MATERIAL}

The Supplementary Material for this article can be found online at: https://www.frontiersin.org/articles/10.3389/fmicb. 2021.741597/full\#supplementary-material

Comeau, D., Novinscak, A., Joly, D. L., and Filion, M. (2020). Spatio-temporal and cultivar-dependent variations in the cannabis microbiome. Front. Microbiol. 11:491. doi: $10.3389 /$ fmicb. 2020.00491

Danziger, N., and Bernstein, N. (2021). Light matters: effect of light spectra on cannabinoid profile and plant development of medical cannabis (Cannabis sativa L.). Industr. Crops Produ. 164:113351. doi: 10.1016/j.indcrop.2021. 113351

Dastogeer, K. M. G., Tumpa, F. H., Sultana, A., Akter, M. A., and Chakraborty, A. (2020). Plant microbiome-an account of the factors that shape community composition and diversity. Curr. Plant Biol. 23:100161. doi: 10.1016/j.cpb.2020. 100161

De Cáceres, M., and Jansen, F. (2019). indicspecies-Package: Studying the Statistical Relationship Between Species and Groups of Sites. R Package, V.1.7.1.

de Jesus Sousa, J. A., and Olivares, F. L. (2016). Plant growth promotion by streptomycetes: ecophysiology, mechanisms and applications. Chem. Biol. Technol. Agric. 3, 1-12.

Delgado-Baquerizo, M., Oliverio, A. M., Brewer, T. E., Benavent-González, A., Eldridge, D. J., Bardgett, R. D., et al. (2018). A global atlas of the dominant bacteria found in soil. Science 359:320. doi: 10.1126/science.aap9516

Figueiredo, M. V. B., Seldin, L., Araujo, F. F., and Mariano, R. L. R. (2010). "Plant growth promoting rhizobacteria: fundamentals and applications," in FPlant Growth and Health Promoting Bacteria. Microbiology Monographs, ed. D. Maheshwari (Berlin: Springer).

Floc'h, J.-B., Hamel, C., Lupwayi, N., Harker, K. N., Hijri, M., and St-Arnaud, M. (2020). Bacterial communities of the canola rhizosphere: network analysis reveals a core bacterium shaping microbial interactions. Front. Microbiol. 11:1587. doi: 10.3389/fmicb.2020.01587

Garbeva, P., Veen, J. V., and Elsas, J. D. V. (2004). Microbial diversity in soil: selection of microbial populations by plant and soil type and implications for disease suppressiveness. Ann. Rev. Phytopathol. 42, 243-270. doi: 10.1146/ annurev.phyto.42.012604.135455

Gautam, A. K., Kant, M., and Thakur, Y. (2013). Isolation of endophytic fungi from cannabis sativa and study their antifungal potential. Arch. Phytopathol. Plant Protect. 46, 627-635.

Harman, G. E., Howell, C. R., Viterbo, A., Chet, I., and Lorito, M. (2004) Trichoderma speciesopportunistic, avirulent plant symbionts. Nat. Rev. Microbiol. 2, 43-56. doi: 10.1038/nrmicro797

Hervé, M., and Hervé, M. M. (2020). Package 'RVAideMemoire'. Available online at: https://cran.r-project.org/web/packages/RVAideMemoire/index.html (accessed September 7, 2021).

Huang, R., Zeng, J., Zhao, D., Cook, K. V., Hambright, K. D., and Yu, Z. (2020). Sediment microbiomes associated with the rhizosphere of emergent macrophytes in a shallow, subtropical lake. Limnol. Oceanogr. 65, S38-S48. 
Kaur, R., Kaur, J., and Singh, R. S. (2011). Nonpathogenic fusarium as a biological control agent. Plant Pathol. J. 9, 79-91.

Korenblum, E., Dong, Y., Szymanski, J., Panda, S., Jozwiak, A., Massalha, H., et al. (2020). Rhizosphere microbiome mediates systemic root metabolite exudation by root-to-root signaling. Proc. Natl. Acad. Sci. U.S.A. 117, 3874-3883. doi: 10.1073/pnas.1912130117

Kurtz, Z. D., Muller, C. L., Miraldi, E. R., Littman, D. R., Blaser, M. J., and Bonneau, R. A. (2015). Sparse and compositionally robust inference of microbial ecological networks. PLoS Comput. Biol. 11:e1004226. doi: 10.1371/journal. pcbi.1004226

Kusari, P., Kusari, S., Spiteller, M., and Kayser, O. (2013). Endophytic fungi harbored in Cannabis sativa L.: diversity and potential as biocontrol agents against host plant-specific phytopathogens. Fungal. Diversity 60, 137-151. doi: 10.1007/s13225-012-0216-3

Law, A. D., Mcnees, C. R., and Moe, L. A. (2020). The microbiology of hemp retting in a controlled environment: steering the hemp microbiome towards more consistent fiber production. Agronomy 10:492. doi: 10.3390/ agronomy10040492

Lay, C.-Y., Bell, T. H., Hamel, C., Harker, K. N., Mohr, R., Greer, C. W., et al. (2018). Canola root-associated microbiomes in the canadian prairies. Front. Microbiol. 9:1188. doi: 10.3389/fmicb.2018.01188

Lebeis, S. L. (2014). The potential for give and take in plant-microbiome relationships. Front. Plant Sci. 5:287. doi: 10.3389/fpls.2014.00287

McKernan, K., Spangler, J., Zhang, L., Tadigotla, V., Helbert, Y., Foss, T., et al. (2016). Cannabis microbiome sequencing reveals several mycotoxic fungi native to dispensary grade cannabis flowers. F1000Research 4:1422. doi: 10 . 12688/f1000research.7507.2

Miao, S., Zhao, H., Pan, S., Zhang, N., and Qiao, Y. (2020). Elevated CO(2) weakens the shift in bacterial community structure in response to 8-year soybean straw return in the same experiment. Int. J. Phytor. 23, 505-510. doi: 10.1080/ 15226514.2020 .1825332

Nilsson, R. H., Larsson, K.-H., Taylor, A. F., Bengtsson-Palme, J., Jeppesen, T. S., Schigel, D., et al. (2018). The UNITE database for molecular identification of fungi: handling dark taxa and parallel taxonomic classifications. Nucleic Acids Res. 47, D259-D264. doi: 10.1093/nar/gky1022

Oksanen, J., Blanchet, F., Friendly, M., Kindt, R., Legendre, P., Mcglinn, D., et al. (2019). vegan: Community Ecology Package. R package version 2.5-6.

Oksanen, J., Blanchet, F., Friendly, M., Kindt, R., Legendre, P., Mcglinn, D., et al. (2020). Package-vegan: Community ecology package. R package version 2.5-6".

Pagnani, G., Pellegrini, M., Galieni, A., D’egidio, S., Matteucci, F., Ricci, A., et al. (2018). Plant growth-promoting rhizobacteria (PGPR) in Cannabis sativa 'finola' cultivation: an alternative fertilization strategy to improve plant growth and quality characteristics. Industr. Crops Products 123, 75-83. doi: 10.1016/j. indcrop.2018.06.033

Pascale, A., Proietti, S., Pantelides, I. S., and Stringlis, I. A. (2019). Modulation of the root microbiome by plant molecules: the basis for targeted disease suppression and plant growth promotion. Front. Plant Sci. 10:1741. doi: 10.3389/fpls.2019. 01741

Peşteanu, A., and Bostan, M. (2020). Perfecţionarea unor elemente tehnologice la producerea materialului săditor pentru fondarea livezilor moderne de măr. Stiinta Agricola 1, 52-59.

Philippot, L., Raaijmakers, J. M., Lemanceau, P., and Van Der Putten, W. H. (2013). Going back to the roots: the microbial ecology of the rhizosphere. Nat. Rev. Microbiol. 11, 789-799. doi: 10.1038/nrmicro3109

Poisot, T., Foster, Z. S. L., Sharpton, T. J., and Grünwald, N. J. (2017). Metacoder: an $\mathrm{R}$ package for visualization and manipulation of community taxonomic diversity data. PLoS Comput. Biol. 13:e1005404. doi: 10.1371/journal.pcbi. 1005404

Punja, Z. K. (2021). Epidemiology of Fusarium oxysporum causing root and crown rot of cannabis (Cannabis sativa L., marijuana) plants in commercial greenhouse production. Can. J. Plant Pathol. 43, 216-235.

Quast, C., Pruesse, E., Yilmaz, P., Gerken, J., Schweer, T., Yarza, P., et al. (2013). The SILVA ribosomal RNA gene database project: improved data processing and web-based tools. Nucleic Acids Res. 41, D590-D596. doi: 10.1093/nar/gks 1219

R Core Team (2020). R: A Language and Environment for Statistical Computing. Version 4.0. 2.

Ryder, L. S., Harris, B. D., Soanes, D. M., Kershaw, M. J., Talbot, N. J., and Thornton, C. R. (2012). Saprotrophic competitiveness and biocontrol fitness of a genetically modified strain of the plant-growth-promoting fungus
Trichoderma hamatum GD12. Microbiology 158, 84-97. doi: 10.1099/mic.0. 051854-0

Saloner, A., and Bernstein, N. (2020). Response of medical cannabis (Cannabis sativa L.) to nitrogen supply under long photoperiod. Front. Plant Sci. 11:1517.

Sapkota, R., Knorr, K., Jørgensen, L. N., O’hanlon, K. A., and Nicolaisen, M. (2015). Host genotype is an important determinant of the cereal phyllosphere mycobiome. New Phytol. 207, 1134-1144. doi: 10.1111/nph.13418

Scott, M., Rani, M., Samsatly, J., Charron, J.-B., and Jabaji, S. (2018). Endophytes of industrial hemp (Cannabis sativa L.) cultivars: identification of culturable bacteria and fungi in leaves, petioles, and seeds. Can. J. Microbiol. 64, 664-680. doi: $10.1139 / \mathrm{cjm}-2018-0108$

Shannon, P., Markiel, A., Ozier, O., Baliga, N. S., Wang, J. T., Ramage, D., et al. (2003). Cytoscape: a software environment for integrated models of biomolecular interaction networks. Genome Res. 13, 2498-2504. doi: 10.1101/ gr.1239303

Singh, B. K., and Trivedi, P. (2017). Microbiome and the future for food and nutrient security. Microbial. Biotechnol. 10, 50-53.

Studholme, D. J., Harris, B., Le Cocq, K., Winsbury, R., Perera, V., Ryder, L., et al. (2013). Investigating the beneficial traits of Trichoderma hamatum GD12 for sustainable agriculture-insights from genomics. Front. Plant Sci. 4:258. doi: $10.3389 /$ fpls.2013.00258

Taghinasab, M., and Jabaji, S. (2020). Cannabis microbiome and the role of endophytes in modulating the production of secondary metabolites: an overview. Microorganisms 8:3. doi: 10.3390/microorganisms 8030355

Toju, H., Tanabe, A. S., Yamamoto, S., and Sato, H. (2012). High-coverage ITS primers for the DNA-based identification of ascomycetes and basidiomycetes in environmental samples. PLoS One 7:e40863. doi: 10.1371/journal.pone. 0040863

Vandenkoornhuyse, P., Quaiser, A., Duhamel, M., Le Van, A., and Dufresne, A. (2015). The importance of the microbiome of the plant holobiont. New Phytol. 206, 1196-1206. doi: 10.1111/nph.13312

Vujanovic, V., Korber, D. R., Vujanovic, S., Vujanovic, J., and Jabaji, S. (2020). Scientific prospects for cannabis-microbiome research to ensure quality and safety of products. Microorganisms 8:2. doi: 10.3390/microorganisms 8020290

Vurukonda, S. S. K. P., Giovanardi, D., and Stefani, E. (2018). Plant growth promoting and biocontrol activity of Streptomyces spp. as endophytes. Int. J. Mol. Sci. 19:952. doi: 10.3390/ijms19040952

Wickham, H., and Wickham, M. H. (2020). Package 'Plyr' [Online]. Available online at: https://cran.r-project.org/web/packages/plyr/plyr.pdf (accessed September 7, 2021).

Winston, M. E., Hampton-Marcell, J., Zarraonaindia, I., Owens, S. M., Moreau, C. S., Gilbert, J. A., et al. (2014). Understanding cultivar-specificity and soil determinants of the cannabis microbiome. PLoS One 9:e99641. doi: 10.1371/ journal.pone.0099641

Zachow, C., Muller, H., Tilcher, R., and Berg, G. (2014). Differences between the rhizosphere microbiome of beta vulgaris ssp. maritima-ancestor of all beet crops-and modern sugar beets. Front. Microbiol. 5:415. doi: 10.3389/fmicb. 2014.00415

Zamioudis, C., and Pieterse, C. M. J. (2011). Modulation of host immunity by beneficial microbes. Mol. Plant Microbe Int. 25, 139-150. doi: 10.1094/mpmi06-11-0179

Zhou, J., Deng, Y., Luo, F., He, Z., Tu, Q., and Zhi, X. (2010). Functional molecular ecological networks. MBio 1:4.

Conflict of Interest: The authors declare that the research was conducted in the absence of any commercial or financial relationships that could be construed as a potential conflict of interest.

Publisher's Note: All claims expressed in this article are solely those of the authors and do not necessarily represent those of their affiliated organizations, or those of the publisher, the editors and the reviewers. Any product that may be evaluated in this article, or claim that may be made by its manufacturer, is not guaranteed or endorsed by the publisher.

Copyright $\odot 2021$ Ahmed, Smart and Hijri. This is an open-access article distributed under the terms of the Creative Commons Attribution License (CC BY). The use, distribution or reproduction in other forums is permitted, provided the original author(s) and the copyright owner(s) are credited and that the original publication in this journal is cited, in accordance with accepted academic practice. No use, distribution or reproduction is permitted which does not comply with these terms. 